Research Article

\title{
Research on Vibration Characteristics of a Ceramic Spindle Based on the Reverse Magnetic Effect
}

\author{
Ke Zhang, Zinan Wang $(\mathbb{D}$, Huaitao Shi, Xiaotian Bai, and Zhan Wang \\ School of Mechanical Engineering, Shenyang Jianzhu University, Shenyang 110168, China \\ Correspondence should be addressed to Zinan Wang; wzn1404589743@126.com
}

Received 18 December 2018; Revised 27 February 2019; Accepted 1 April 2019; Published 2 May 2019

Academic Editor: Huu-Tai Thai

Copyright (c) 2019 Ke Zhang et al. This is an open access article distributed under the Creative Commons Attribution License, which permits unrestricted use, distribution, and reproduction in any medium, provided the original work is properly cited.

\begin{abstract}
The electromagnetic radial force about a ceramic spindle affects the spindle dynamic, which determines the quality of processing. Using a Timoshenko beam unit to build the dynamic model for the ceramic spindle, the dynamic characteristic of an angular contact ball bearing is analyzed using a nonlinear bearing model. The electromagnetic magnetization model was established based on Maxwell's theory to calculate electromagnetic magnetic density and radial force. The influence about the reverse magnetic field characteristic of the ceramic rotating shaft and dynamic stiffness of the contact ball bearing on the dynamic phenomena of the spindle is analyzed, which is verified by experiments. The results show that the magnetic effect produced by the reverse magnetic of ceramic rotation shaft has a great influence on the electromagnetic radial force. Compared with the paramagnetic effect of the metal shaft, the dynamic characteristics of the spindle can be significantly improved. Considering the coupling relationship between the radial force of the magnetic field and the bearing contact force, dynamic stiffness, and other factors, the accuracy of the model simulation is highly consistent with the test results. In particular, the ceramic spindle model has been successful in predicting with high accuracy and is suitable for multiple extreme working conditions. The parameters, such as initial eccentricity of the rotor, bearing preload, and rotating speed, can be adjusted to restrain the vibration of spindle. The ceramic spindle model provides a theoretical basis for the dynamics development of a high-speed spindle.
\end{abstract}

\section{Introduction}

High-speed machine tools play an important role in today's manufacturing industries and can significantly improve product surface quality and machining accuracy [1-3]. Due to the ceramic materials characteristics of high stiffness, small deformation, and reverse magnetic, in special extreme environments such as Aerospace area (ultrahigh temperature and ultralow temperature), high corrosion area, and high requirement for magnetic area (ultralow magnetic field), the performance of ceramic spindle is better than metal spindle. However, the vibration of the ceramic spindle is large in these extreme environments. It is of great significance to study the dynamic performance of the highspeed ceramic spindle.

Considerable research studies related to improve a more efficient machine tool, energy savings, and vibration avoidance of the spindle have been published. The purpose of modeling the spindle is to find the shaft deformation, bearing characteristics, and magnetic effect which have the greatest effect on the static and dynamic properties of the spindle systems. Lee and Hoang [4] studied the consideration of the continuous mass effects, eccentricity, and rotational inertia give a more realistic model that was established through the Timoshenko beam for spindle system and investigated the coupled lateral and torsional effects on the system vibration behavior. However, the model of An-Chen Lee neglects the radial force on the shaft. Zivkovic et al.'s model [5] is based on the mechanical model of the bearing and the numerical model FEM (Finite Element Method) of the spindle to predict bearing characteristics and the change in static stiffness of the spindle accuracy. Different speed responds are verified by experiments. But the 
dynamic stiffness is not mentioned in the model. Hu et al. [6] studied the effects of preload and preload method on the rotational performance of the spindle-bearing system under different speeds. However, the paper is not to solve the coupled problems of the spindle-bearing systems. Hong et al. [7] described a kinematic model of a five-axis machine tool under position-independent and positiondependent geometric errors associated with rotary axes through the experiments to verify the enlargement of a periodic radial error motion of $\mathrm{C}$-axis with $\mathrm{B}$-axis rotation. The model can not perform the vibration accurately in some extreme cases. Some papers [8-10] proposed speed-dependent FRF (Frequency Response Function) spindle model. The coupled spindle model based on Timoshenko beam can be predicted. But the predicted models are not better adapted to the high stiffness of the ceramic spindle.

Vyroubal [11] presented a method focused on compensation of machine's thermal deformation in spindle axis direction based on decomposition analysis that introduces cheap and effective strategy how to compensate machine tool thermal deformation to reduce the residual thermal deformation greatly. Yang et al. [12] proposed least square support vector machine LS-SVM (Least Square Support Vector Machine) to establish axial elongation and radial thermal yaw about motorized spindle thermal error model which results demonstrated that the axial (in the $X$ and $Y$ directions) and radial accuracies were improved greatly through experiments. Both the papers did not consider the small deformation of the ceramic materials. Grossi [13] studied the specific cutting force coefficients in the entire speed range by a single milling test through aluminum coefficients traditional approach to validate the efficiency and effectiveness of the proposed technique, which is not adaptive in high-temperature environment. Werner [14] employed the electromagnetic model with an electromagnetic moment to calculate radial and angular electromagnetic stiffnesses, the mass moment of inertia, and the gyroscopic effect of the rotor, but magnetic radical force is not considered. Liu et al. [15] proposed a model including Hertz contact force and unbalanced electromagnetic force to study the dynamic properties of the spindle system. The studies [16-18] developed Maxwell stress tensor nonlinear harmonic methods and evaluated the vibration influence caused by UMP (Unbalanced Magnetic Pull). However, the magnet characteristics of different materials were ignored. Some traditional metal dynamic models could not be better suited for the ceramic spindle.

However, Oliver et al., Mironov et al., and Khazdozian et al. [19-21] have analyzed some magnetic characteristics of ceramic materials and reverse magnetic effect on the ceramic spindle, and bearing has not been investigated. In this paper, we give a general method that considers reverse magnetic effect that can be used to predict the vibration characteristics of the ceramic spindle systems. Based on the application of Timoshenko beam unit, a dynamic model is established in this paper.
The relation between the dynamic stiffness, gyroscopic moment, and centrifugal force change is analyzed using the bearing raceway control theory. The relationship between the magnetic density and electromagnetic radial force of ceramic materials is studied based on Maxwell's theory. The radial force of the reverse magnetic and paramagnetic effects and the contact force of bearing Hertz on the dynamics of spindle are discussed. The complex dynamic mechanism of a high-speed ceramic spindle system is revealed through a ceramic spindle model simulation and multimodal test. The ceramic spindle model can predict the magnetic field and dynamic characteristics well in some special extreme situations.

\section{Dynamic Model of a High-Speed Ceramic Spindle}

2.1. Spindle System Beam Element Model. Ceramic spindle bearings are installed on the ceramic shaft. A Timoshenko beam element is used to establish the dynamic model. The rotating shaft of the ceramic spindle is equivalent to stimulate vibration in the beam under the action of multiple factors, such as centrifugal force of the bearing and electromagnetic radial force [22]. The model uses the Hamiltonian principle to calculate the kinetic energy, potential energy, and damping work of the beam element. According to the damping and stiffness matrix, the influence of the electromagnetic radial force and bearing contact stress of the spindle system vibration is obtained. The discrete model of the ceramic spindle beam element is shown in Figure 1. The front bearing and rear bearing are equivalent to a spring and damping, which are $K_{n 1}, C_{n 1}$ and $K_{n 2}, C_{n 2}$, respectively.

The discrete model assumes that the $x y z$ axis is the inertial reference frame. Every axis unit is equivalent to 5 degrees of freedom: three directions of translational freedom are $x, y$, and $z(u, v, w)$ and the degrees of freedom of rotation around the $y$ and $z$ axes $\left(\theta_{z}\right.$ and $\left.\theta_{y}\right)$. Beam element nodes can be represented by point $\mathrm{P}$ in ball coordinates, $\varphi$ is the point $\mathrm{P}$ which rotated about the axis of rotation, and $d_{\mathrm{T}}$ is the distance between the point $\mathrm{P}$ and the center of the section circle. The spindle model of Timoshenko beam is shown in Figure 2. The displacement of point $\mathrm{P}$ is represented by $q, q=\left(u, v, w, \theta_{z}, \theta_{y}\right)^{T}$. The point $\mathrm{P}$ coordinate is

$$
\left\{\begin{array}{l}
x \\
y \\
z
\end{array}\right\}=\left\{\begin{array}{c}
u-r \cos \varphi \sin \theta_{z}+r \sin \varphi \sin \theta_{y} \\
v+r \cos \varphi \cos \theta_{z} \\
w+r \sin \varphi \cos \theta_{y}
\end{array}\right\} .
$$

Point $\mathrm{P}$ generates kinetic energy, strain energy, and external force work as the beam element changes between the instantaneous $t_{1}$ and $t_{2}$ moments. $T$ and $V$ are, respectively, shown in Figures 2(a) and 2(b). According to Hamilton's principle, formula (2) is derived similarly to the one reported in [23]: 


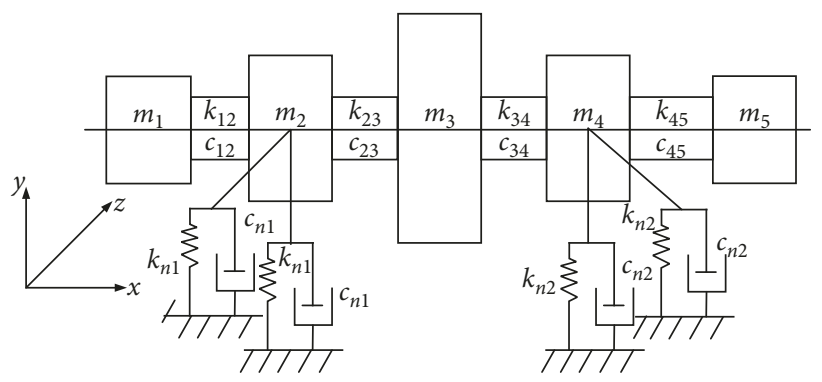

Figure 1: Discrete model of the ceramic spindle beam element.

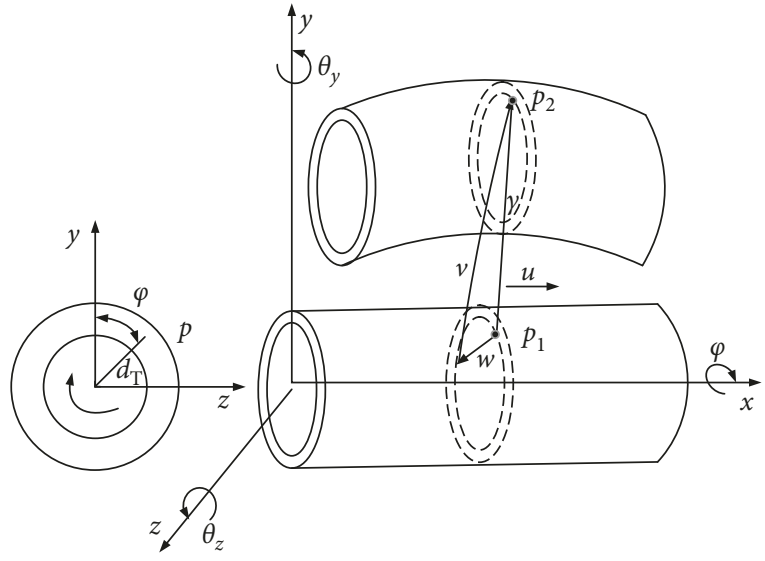

(a)

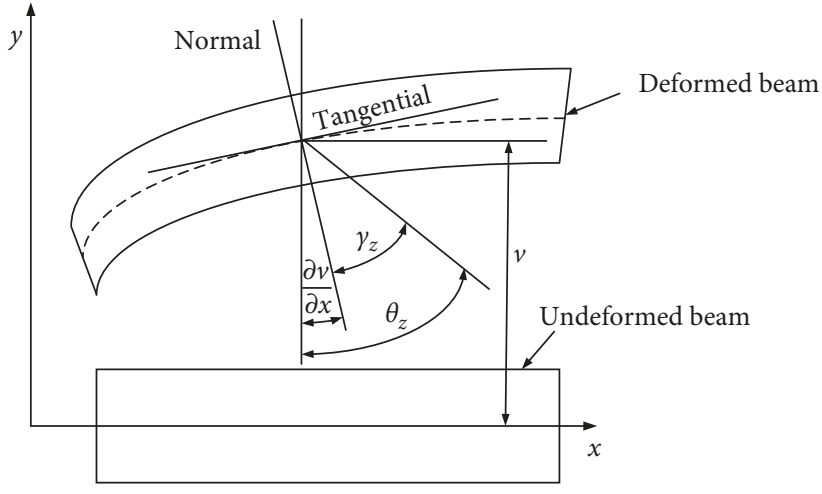

(b)

Figure 2: Spindle model of Timoshenko beam: (a) kinetic energy; (b) shaft shear deformation.

$$
\begin{aligned}
\delta I= & \delta \int_{t_{1}}^{t_{2}}(T-V+W) d t=0 \\
T= & \frac{1}{2} \int_{0}^{L} J_{D} \Omega^{2} d x+\int_{0}^{L} \frac{1}{2}\left(\dot{u}^{2}+\dot{v}^{2}+\dot{w}^{2}\right) d x \\
& +\frac{1}{2} \int_{0}^{L} I_{D} \rho\left(\dot{\theta}_{y}^{2}+\dot{\theta}_{z}^{2}\right) d x+\frac{1}{2} \int_{0}^{L} \Omega \rho J_{D}\left(\dot{\theta}_{y} \theta_{z}-\theta_{y} \dot{\theta}_{z}\right) d x \\
V= & \frac{1}{2} \int_{0}^{L} E I\left[\left(\frac{\partial u}{\partial x}\right)^{2}+\left(\frac{\partial v}{\partial x}\right)^{2}\right] d x \\
& +\frac{1}{2} K_{s} G S\left[\left(\frac{\partial v}{\partial x}+\theta_{Z}\right)^{2}+\left(\frac{\partial w}{\partial x}+\theta_{y}\right)^{2}\right] \\
& +\frac{1}{2} \int_{0}^{L} F_{\mathrm{a}}\left[\left(\frac{\partial v}{\partial x}\right)^{2}+\left(\frac{\partial w}{\partial x}\right)^{2}\right] d x, \\
W= & \frac{1}{2} \int_{0}^{L} \Omega^{2}\left(v^{2}+w^{2}\right) \rho S d x+\int_{0}^{L}\left(m_{y} \theta_{y}+m_{z} \theta_{z}\right) d x
\end{aligned}
$$

where $I_{D}$ is the inertia moment, $I_{D}=(1 / 4) m_{D}\left(b^{2}-a^{2}\right)$, $J_{D}$ is the moment of inertia, $J_{D}=(1 / 2) m_{D}\left(b^{2}-a^{2}\right), \theta_{z}$ is the rotation angle of the $x-y$ section, $\theta_{z}=\gamma_{z}+(\partial v / \partial x)$, and $\theta_{y}$ is the rotation angle of the $x-z$ section, $\theta_{y}=\gamma_{y}-(\partial \omega / \partial x)$.

Considering the formulas of motion of each axis, the dynamic model of the spindle is obtained:

$$
\left[M_{\mathrm{d}}+M\right]\{\ddot{q}\}+n[C]\{\dot{q}\}+[K]\{q\}=F(t) .
$$

2.2. High-Speed Ceramic Angle Contact Bearing Model. In the process of high-speed rotation, the spindle produces Hertz contact force, which will affect the dynamic characteristics of the spindle. The bearing is mainly composed of an outer ring, inner ring, ball, and retainer. The bearing structure diagram is shown in Figure 3. In the process of bearing high-speed rotation, the ball load will change with angle displacement change of the inner and outer rings. The dynamic stiffness of the bearing changes accordingly. The Jones raceway bearing model is used to study the instantaneous motion of the bearing under steady running as the main research object. The bearing outer ring is fixed in the bearing housing by a preload. Assuming that the outer ring and the center of outer ring groove curvature are fixed, the ball and the center of the inner ring groove curvature move relatively. The geometric bearing deformation model is established to analyze the ball and the center of the inner ring groove curvature transient position change where the ball $j$ is in $\varphi_{j}$ position under steady motion.

$$
\varphi_{j}=\frac{2 \pi(j-1)}{Z} .
$$

The contact stress between the ball and the inner ring is nonlinear. According to the Hertz contact theory, the ball contact point inner surface with the bearing inner and outer ring curvature center is positive and that with the outer 


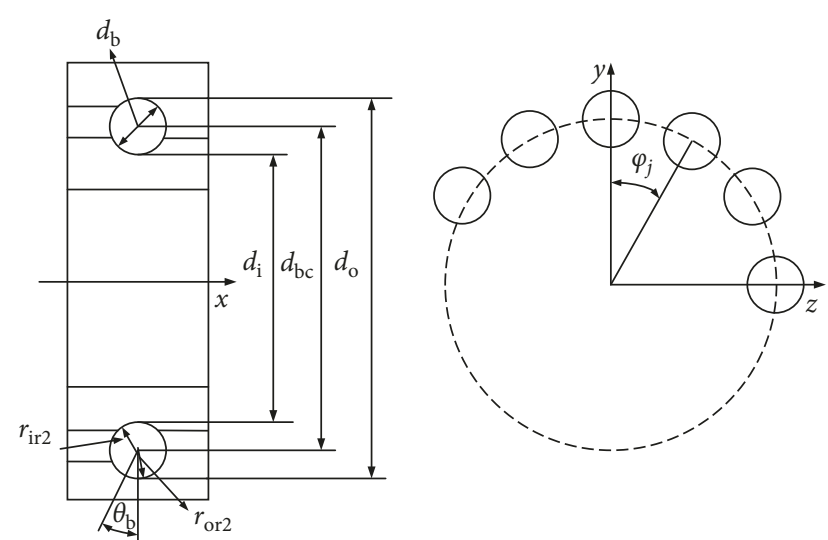

FIGURE 3: Bearing structure.

surface is negative. The total curvature of the angle contact ball bearing inner and outer rings with the contact point of the rolling body, respectively, is

$$
\left\{\begin{array}{l}
\sum \rho_{\mathrm{i}}=\rho_{\mathrm{b} 1}+\rho_{\mathrm{b} 2}+\rho_{\mathrm{ir} 1}+\rho_{\mathrm{ir} 2} \\
\sum \rho_{\mathrm{o}}=\rho_{\mathrm{b} 1}+\rho_{\mathrm{b} 2}+\rho_{\mathrm{or} 1}+\rho_{\mathrm{or} 2}
\end{array}\right.
$$

where $\sum \rho_{\mathrm{i}}$ and $\sum \rho_{\mathrm{o}}$ are the total curvature of the bearing inner and outer rings with the contact point of the ball; $\rho_{\text {irl }}$ and $\rho_{\text {orl }}$ are the normal curvature of the bearing inner and outer rings with the contact point of the ball, $\rho_{\text {ir } 1}=2 \cos \theta /\left(d_{\mathrm{bc}}-d_{\mathrm{i}}\right)$ and $\rho_{\text {or } 1}=2 \cos \theta /\left(d_{\mathrm{o}}-d_{\mathrm{bc}}\right) ; \rho_{\text {ir } 2}$ and $\rho_{\text {or2 }}$ are the tangential curvature of the bearing inner and outer rings with the contact point of the ball, $\rho_{\mathrm{ir} 2}=-1 / r_{\mathrm{ir}}$, $\rho_{\text {or } 2}=-1 / r_{\mathrm{or}}$; and $\rho_{\mathrm{b} 1}, \rho_{\mathrm{b} 2}$ are the normal and tangential curvature of bearing with the contact point of the ball, $\rho_{\mathrm{b} 1}=\rho_{\mathrm{b} 2}=2 / d_{\mathrm{b}}$ :

$$
\left\{\begin{array}{l}
K_{\mathrm{ik}}=\frac{1.88}{\delta_{\mathrm{ik}}^{3 / 2} \sqrt{\sum \rho_{\mathrm{i}}}}\left[\frac{1-v_{\mathrm{b}}^{2}}{E_{\mathrm{b}}}+\frac{1-v_{\mathrm{i}}^{2}}{E_{\mathrm{i}}}\right]^{-1}, \\
K_{\mathrm{ok}}=\frac{1.88}{\delta_{\mathrm{ok}}^{3 / 2} \sqrt{\sum \rho_{\mathrm{o}}}}\left[\frac{1-v_{\mathrm{b}}^{2}}{E_{\mathrm{b}}}+\frac{1-v_{\mathrm{o}}^{2}}{E_{\mathrm{o}}}\right]^{-1} \\
\delta=\delta_{\mathrm{ik}}+\delta_{\mathrm{ok}}=\left(\frac{\mathrm{Q}_{\mathrm{ik}}}{K_{\mathrm{ik}}}\right)^{2 / 3}+\left(\frac{\mathrm{Q}_{\mathrm{ok}}}{K_{\mathrm{ok}}}\right)^{2 / 3} .
\end{array}\right.
$$

Bearing friction is produced by centrifugal force acting on the ball at high speed. The load and displacement of bearing under combined loading are shown in Figure 4. Figure 4(a) clearly shows that the gyroscopic moment can balance the friction generated between the ball and the contact of the inner ring and outer ring raceway surface. Figure 4(b) reflects the relative displacement relation of inner and outer rings under the conditions of some force and gyroscopic moment. According to the Jones raceway control theory, ball balance formula (8) is derived similarly to the one reported in [24].

$$
\left\{\begin{array}{l}
M_{\mathrm{jk}}=J \omega_{\mathrm{i}}^{2}\left(\frac{\omega_{\mathrm{b}}}{\omega_{\mathrm{i}}}\right)_{j}\left(\frac{\omega_{\mathrm{p}}}{\omega_{\mathrm{i}}}\right)_{j} \sin \beta, \\
Q_{\mathrm{o} j} \cos \theta_{\mathrm{b}}-\frac{M_{\mathrm{jk}}}{D}\left(\lambda_{\mathrm{o} j} \sin \theta_{\mathrm{b}}-\lambda_{\mathrm{i} j} \sin \theta_{\mathrm{i}}\right)-Q_{\mathrm{ik}} \cos \theta_{\mathrm{i}}=F_{\mathrm{c} j}, \\
Q_{\mathrm{o} j} \sin \theta_{\mathrm{b}}-\frac{M_{\mathrm{jk}}}{D}\left(\lambda_{\mathrm{i} j} \cos \theta_{\mathrm{i}}-\lambda_{\mathrm{o} j} \cos \theta_{\mathrm{b}}\right)-Q_{\mathrm{i} j} \sin \theta_{\mathrm{i}}=0 .
\end{array}\right.
$$

The bearing inner ring under internal load is in a state of balance, and the bearing inner ring balance formula is

$$
\left\{\begin{array}{l}
F_{z \mathrm{a}}-\sum_{j=1}^{j=Z}\left(Q_{\mathrm{ik}} \sin \theta_{\mathrm{i}}-\frac{M_{\mathrm{jk}}}{D} \cos \theta_{\mathrm{i}}\right)=0, \\
F_{z \mathrm{r}}-\sum_{j=1}^{j=Z}\left(Q_{\mathrm{ik}} \cos \theta_{\mathrm{i}} \sin \varphi_{j}-\frac{M_{\mathrm{jk}}}{D} \sin \theta_{\mathrm{i}} \sin \varphi_{j}\right)=0, \\
M_{z}-\sum_{j=1}^{j=Z}\left(Q_{\mathrm{ik}} \sin \theta_{\mathrm{i}} \cos \varphi_{j}+\frac{M_{\mathrm{jk}}}{D} \sin \theta_{\mathrm{i}} \cos \varphi_{j}\right) \\
\cdot\left(0.5 D+\left(f_{\mathrm{i}}-0.5\right) D \cos \varphi_{j}\right)=0, \\
F_{\mathrm{ap}}=1.2 \tan \theta F_{z \mathrm{r}}+F_{z \mathrm{a}} \tan \beta,
\end{array}\right.
$$

where $F_{c j}$ is the centrifugal force of ball, $F_{\mathrm{c} j}=(1 / 2) m D_{m} \omega_{\mathrm{i}}^{2}\left(\omega_{\mathrm{p}} / \omega_{\mathrm{i}}\right)_{j}^{2}$.

2.3. Magnetization Model of High-Speed Ceramic Spindle Model. The stator of spindle is hot-pressed on the shell. The rotor is driven by a variable frequency magnetic field to rotate at high speed. The magnetic potential distribution of spindle stator and rotor is shown in Figure 5. The two points $\alpha, \beta$ produce the $z$ point magnetic potential.

$$
\left\{\begin{array}{l}
A_{z}=A_{\alpha}+A_{\beta}, \\
A_{z}=\frac{\mu_{0} i}{\pi} \sum_{1}^{m} \frac{1}{m} \frac{R_{\mathrm{s}}^{m}}{d_{\mathrm{c}}^{m}}\left(\frac{r_{\mathrm{r}}^{2 m}+d_{\mathrm{c}}^{2 m}}{R_{\mathrm{s}}^{2 m}-r_{\mathrm{r}}^{2 m}}\right)\left(\frac{d_{\mathrm{c}}^{m}}{R_{\mathrm{s}}^{m}}+\frac{R_{\mathrm{s}}^{m}}{r_{\mathrm{r}}^{m}}\right) k_{\mathrm{pm}} \sin m \xi,
\end{array}\right.
$$

where $k_{\mathrm{pm}}$ is the pitch factor of $m$ harmonic $k_{\mathrm{pm}}=\sin m(y / \tau) 90^{\circ}$.

Air gap tangential magnetic field density $B_{\theta}$ is given by

$$
B_{\theta}=-\frac{\mu_{0} i}{\pi} \sum_{1}^{\infty} \frac{r_{\mathrm{r}}^{m}}{d_{\mathrm{c}}^{m}}\left(\frac{R_{\mathrm{s}}^{2 m}+d_{\mathrm{c}}^{2 m}}{R_{\mathrm{s}}^{2 m}-r_{\mathrm{r}}^{2 m}}\right)\left(\frac{r_{\mathrm{r}}^{m}}{R_{\mathrm{s}}^{m}}-\frac{R_{\mathrm{s}}^{m}}{r_{\mathrm{r}}^{m}}\right) k_{\mathrm{pm}} \sin m \theta .
$$

It can be seen on the surface of the stationary rotor that the spindle magnetic field is orthogonal to the stator and 


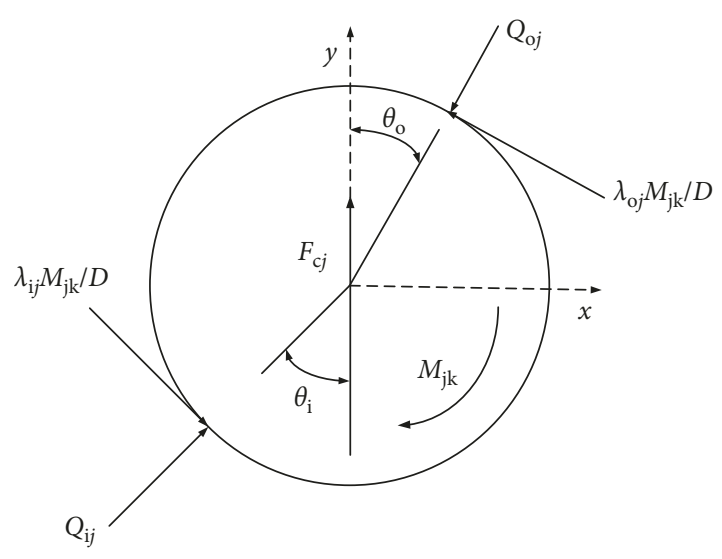

(a)

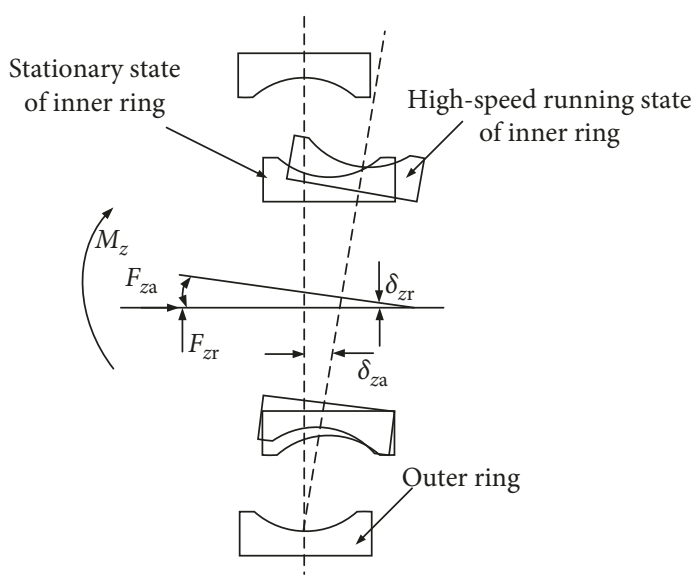

(b)

FIGURE 4: Load and displacement of bearing under combined loading: (a) ball load; (b) the relative displacement relation of inner and outer ring.

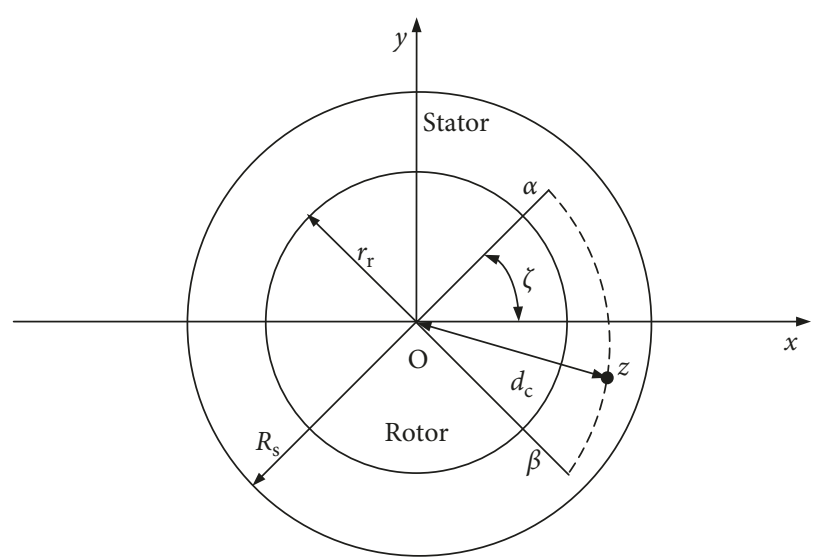

FIGURE 5: Magnetic potential distribution of spindle stator and rotor.

rotor surface. Even if eccentricity occurs, its value is ignored. The spindle coil is placed on the stator, and the air gap density of the radial magnetic field is approximated by the Fourier transform:

$$
\left\{\begin{array}{l}
B_{\mathrm{r} q}=\frac{\mu_{0} i}{\pi} \sum_{1}^{m} \frac{r_{\mathrm{r}}^{m}}{d_{\mathrm{c}}^{m}}\left(\frac{R_{\mathrm{s}}^{2 m}+d_{\mathrm{c}}^{2 m}}{R_{\mathrm{s}}^{2 m}-r_{\mathrm{s}}^{2 m}}\right)\left(\frac{r_{\mathrm{r}}^{m}}{R_{\mathrm{s}}^{m}}+\frac{R_{\mathrm{s}}^{m}}{r_{\mathrm{r}}^{m}}\right) k_{\mathrm{pm}} \cos m \theta \\
=\frac{2 \mu_{0} i}{\pi \delta} \sum_{1}^{m} \frac{1}{m} k_{\mathrm{pm}} \cos m \theta \\
H=\frac{1}{\mu_{0}} B_{\mathrm{r} q}-M_{\mathrm{e}} \\
B_{\mathrm{m}}=\mu_{0}\left(1+\chi_{\mathrm{m}}\right) H=\mu_{0} \mu_{\mathrm{r}} H
\end{array}\right.
$$

where $\chi_{\mathrm{m}}$ is the medium of magnetic susceptibility; $\chi_{\mathrm{c}}$ is the ceramic magnetic susceptibility $10^{-5} \mathrm{~cm}^{3} / \mathrm{mol} ; \chi_{\mathrm{Fe}}$ is the steel magnetic susceptibility $14000 \mathrm{~cm}^{3} / \mathrm{mol} ; \mu_{\mathrm{r}}$ is the relative permeability; and $\mu_{0}$ is the air permeability.

The stator and rotor magnetic media exist from Ampere's loop theorem:

$$
\oint_{L} H \cdot d r=\left(H_{2}-H_{1}\right) \cdot \vec{t} \Delta l=\oiint_{S} j \cdot d S,
$$

where $H_{1}$ is the air magnetic field intensity vector; $H_{2}$ is the ceramic magnetic field intensity vector; and $S$ is the stator and rotor contact area, $S=2 \pi r \Delta l ; \vec{t}$ is the tangential unit vector; and $\Delta l$ is the length of $\mathrm{AB}, \mathrm{AB}=\mathrm{CD}$.

The tangential components of the air gap magnetic density on both sides are continuous. Therefore, the magnetic field density after magnetization $B_{1}$ has a linear relation:

$$
B_{1}=B_{\mathrm{rq}}+B_{\mathrm{m}}
$$

The initial static eccentricity is caused by installation error and stator deformation of the spindle structure. Electromagnetic vibration is one of the main factors of spindle vibration. The actual eccentricity is mainly composed of initial static eccentricity and vibration eccentricity $[25,26]$. The rotor is eccentric relative to the stator and an eccentricity diagram of the spindle is shown in Figure 6. The formula (15) is derived similarly to the one reported in [27].

$$
\delta=\delta_{0}-d_{\mathrm{rq}} \cos (\theta-\gamma)
$$

The air gap $\delta$ varies with the angle $\gamma$. As the air gap is not uniform, the air gap magnetic permeability will change periodically with rotor rotation to generate additional harmonic magnetic fields. Harmonic magnetic fields interact to produce low-order electromagnetic radial forces [28]. According to the Maxwell principle, the effect of an air gap magnetic field on a spindle stator inner surface produces a radial electromagnetic force, which is linear with the square of air gap magnetic field density. The radial electromagnetic force of the spindle $F_{\mathrm{ra}}(\theta, t)$ is derived similarly to one reported in [29]. 


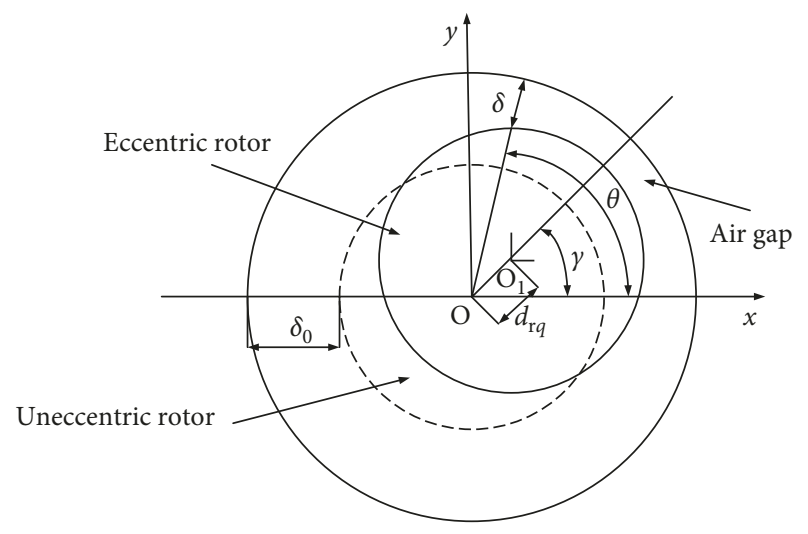

FIGURE 6: Eccentricity diagram of spindle.

$$
F_{\mathrm{r} q}(\theta, t)=\frac{B^{2}(\theta, t)}{2 \mu_{0}}
$$

The spindle air gap magnetic density is

$$
B(\theta, t)=f(\theta, t) \lambda(\theta, t),
$$

where $B(\theta, t)$ is the air gap magnetic density, $\lambda(\theta, t)$ is the air gap magnetic permeance, and $f(\theta, t)$ is the air gap magnetic potential.

2.3.1. Synthetic Potential of Stator and Rotor $f(\theta, t)$. When spindle is running at high speed, the gap magnetic potential is directly related to the spindle current, pole logarithm, and number of stator and rotor slots. The synthetic potential of the stator and rotor is

$$
f(\theta, t)=f_{0}(\theta, t)+\sum_{v} f_{v}(\theta, t)+\sum_{u} f_{u}(\theta, t),
$$

where $f_{0}(\theta, t)$ is the fundamental wave magnetic potential, $f_{v}(\theta, t)$ is the stator harmonic magnetic potential, and $f_{u}(\theta, t)$ is the rotor harmonic magnetic potential.

Considering the bearing damping effect of the rotor system, the fundamental wave synthesis magnetic potential, stator winding harmonic magnetic potential, and rotor winding harmonic magnetic potential are obtained.

$$
\left\{\begin{array}{l}
f_{0}(\theta, t)=1.35 \frac{W_{s} K_{\mathrm{dp} 0}}{p} I_{0} \cos \left(p \theta-\omega_{v} t-\varphi_{0}\right), \\
f_{v}(\theta, t)=1.35 \frac{W_{s} K_{\mathrm{dp} 0}}{v} I_{1} D_{2 v} \cos \left(v \theta-\omega_{v} t-\varphi_{v}\right), \\
f_{u}(\theta, t)=1.35 \frac{W_{s} K_{\mathrm{dp} 0}}{u} I_{2} \cos \left(u \theta-\omega_{u} t-\varphi_{u}\right),
\end{array}\right.
$$

where $I_{\mathrm{s}}$ is the stator current, $I_{\mathrm{r}}$ is the rotor current, and $I_{0}$ is the synthesis current, $I_{0}=I_{\mathrm{s}}+I_{\mathrm{r}}$.

2.3.2. Air Gap Permeance $\lambda(\theta, t)$. The spindle stator and rotor are provided with different number of tooth slots and tooth forms. $\sum_{k_{1}} \sum_{k_{2}} \lambda_{k_{1} k_{2}}$ is much smaller than $\sum_{k_{1}} \lambda_{k_{1}}$ and $\sum_{k_{2}} \lambda_{k_{2}}$, $\sum_{k_{1}} \sum_{k_{2}} \lambda_{k_{1} k_{2}}$ can be neglected. The air gap permeance is equivalent to

$$
\begin{aligned}
\lambda(\theta, t) & =\Lambda_{0}+\sum_{k_{1}} \lambda_{k_{1}}+\sum_{k_{2}} \lambda_{k_{2}}+\sum_{k_{1}} \sum_{k_{2}} \lambda_{k_{1} k_{2}} \\
& \approx \Lambda_{0}+\sum_{k_{1}} \lambda_{k_{1}}+\sum_{k_{2}} \lambda_{k_{2}},
\end{aligned}
$$

where $\Lambda_{0}$ is the fixed part of air gap permeance, $\sum_{k_{1}} \lambda_{k_{1}}$ is the air gap harmonic magnetic permeability caused by stator slotting, $\sum_{k_{2}} \lambda_{k_{2}}$ is the air gap harmonic magnetic permeability caused by rotor slotting, $\sum_{k_{1}} \sum_{k_{2}} \lambda_{k_{1} k_{2}}$ is the air gap harmonic magnetic permeability caused by both stator and rotor slotting interaction.

The air gap permeability is affected by the shape, number of tooth slots, concentricity, and symmetry of the stator and rotor. The gap permeability is expanded by Fourier transform, keeping the first two items in the decomposition item:

$$
\begin{aligned}
\lambda_{k}(\theta, t) & =\frac{\lambda(\theta, t)}{\delta}=\frac{\lambda(\theta, t)}{\delta_{\mathrm{m}}(1-\varepsilon \cos \alpha)} \\
& =\lambda(\theta, t)\left(\lambda_{0}+\lambda_{1} \cos \alpha+\lambda_{2} \cos 2 \alpha+\cdots\right) \\
& \approx \lambda(\theta, t)\left(\frac{1}{\sqrt{1-\varepsilon^{2}}}+\frac{2\left(1-\sqrt{1-\varepsilon^{2}}\right)}{\varepsilon \sqrt{1-\varepsilon^{2}}} \cos \alpha\right) .
\end{aligned}
$$

The stator and rotor gap magnetic densities ignore factors such as a full magnetic circuit, and the slot affects their interaction with each other. Substituting formulas (18) and (20) in formula (17) obtains

$$
\begin{aligned}
B(\theta, t)= & f_{v}(\theta, t) \lambda(\theta, t)+f_{u}(\theta, t) \lambda(\theta, t) \\
\approx & \left(1+\frac{\varepsilon}{2}\right) B_{v} \cos \left(p \theta-\omega_{v} t-\varphi_{0}\right) \\
& +2\left(1+\frac{\varepsilon}{2}\right) \sum_{u} B_{u} \cos \left(p \theta-\omega_{u} t-\varphi_{u}\right),
\end{aligned}
$$

where $\varepsilon$ is the relative eccentricity, $\varepsilon=d_{\mathrm{r} q} / R_{\mathrm{s}}-r_{\mathrm{r}}$.

Substituting formula (22) in formula (16) gives the radial force of spindle:

$$
\begin{aligned}
F(\theta, t) \approx & \frac{1}{2 \mu_{0}}(1+\varepsilon)\left(\frac{B_{1}^{2}}{2} \cos \left(2 p-2 \omega_{v} t-2 \varphi_{0}\right)\right) \\
& +2 \sum_{u} \sum_{v} B_{u} B_{v} \cos \left\{(v \pm u) \theta-\left(\omega_{v} \pm \omega_{u}\right) t+\left(\varphi_{0} \pm \varphi_{u}\right)\right\} .
\end{aligned}
$$

\section{Analysis of Dynamic Influencing Factors of the Spindle System}

Bearing Hertz contact force and electromagnetic radial force will be produced during high-speed rotation of the spindle. To study the effects of the reverse magnetic effect and bearing Hertz contact force on spindle dynamics, we combine every shaft segment of the Timoshenko beam unit with the bearing motion formulas and magnetic field formulas of spindle. Formulas (9) and (23) are substituted in formula (4) to obtain the dynamic formula (24) under the 
action of the bearing contact force and electromagnetic radial force. The dynamic formula of the spindle is solved by an iterative method. Formulas (9), (23), and (24) of the ceramic spindle model show that there is a direct relationship between the vibration and speed of the spindle, centrifugal force of the bearing, pretension force, gyroscopic moment, air gap, electromagnetic radial force, and magnetic field density. The structural parameters of spindle and the length of the rotating shaft discrete system, respectively, are shown in Tables 1 and 2.

$$
\left[M_{\mathrm{d}}+M\right]\{\ddot{q}\}+n[C]\{\dot{q}\}+\left[K_{\mathrm{d}}+K\right]\{q\}=F(t),
$$

where $M_{\mathrm{d}}$ is the element rotational mass matrix, $M$ is the element translational mass matrix, $n$ is the number of shafts, $K_{\mathrm{d}}$ is the element dynamic stiffness matrix, and $F(t)$ is the spindle distribution force vector that considers electromagnetic radial force and bearing contact force.

\subsection{Influence of Electromagnetic Radial Force on the Spindle.} Consider that the rotor rotates at a high speed in the stator, according to the electromagnetic model of the spindle. The stator and rotor are symmetrically distributed, so $1 / 4$ is taken to analyze the electromagnetic field of the spindle. The conditions of the simulations are that the shell of the ceramic spindle is $170 \mathrm{~mm}$, the air gap of stator and rotor is $0.3 \mathrm{~mm}$, the motor pole logarithm is 4 , the inner diameter of the stator is $80 \mathrm{~mm}$, the outer diameter of the rotor is $79.4 \mathrm{~mm}$, and bearing preload force is $400 \mathrm{~N}$. The speed range, for which each increment is $1000 \mathrm{rpm}$, is from $9000 \mathrm{rpm}$ to $18000 \mathrm{rpm}$. The magnetic density of the ceramic and metal spindle is shown in Figure 7. The radial force of the ceramic and metal spindle is shown in Figure 8. Figure 7 shows that the magnetic density of the spindle fluctuates with time. The main reason for this is that the spindle cuts the magnetic induction line under the highspeed frequency converter to form an eddy current. The magnetic force line is bent to form a closed magnetic circuit. The magnetic force line is limited by the air gap magnetic density. The magnetic pole reaches the maximum saturation state. The magnetic pole is affected by the saturation state to show the skin effect and adjacent effect. There is a phenomenon of demagnetization that the current movement in the stator tends to the radial direction of the outer circle in the stator.

The magnetic density fluctuation degree of the ceramic spindle is far less than the metal spindle. Comparing the $\mathrm{ZrO}_{2}$ ceramic spindle with the $40 \mathrm{Cr}$ metal spindle, the ceramic materials are the reverse of the magnetic materials. A ceramic material's permeability magnetic is only $10^{-12}$, which is much smaller than that of a metal material. The ceramic magnetic permeability is too low, and there is no magnetic force line passing through the ceramic spindle rotor. The magnetic force line inside the stator and rotor decreases so that the diffusion flux is suppressed. The air gap magnetic potential produces demagnetization effect to reduce eddy current loss. However, due to the high permeability of metal $40 \mathrm{Cr}$ rotor, under the magnetization effect, the internal molecules in the magnetic medium can be
TABLE 1: The structural parameters of spindle.

\begin{tabular}{lcc}
\hline \multicolumn{2}{c}{ Stator and rotor } \\
\hline 1 & Number of stator slots, $Z_{1}$ & 24 \\
2 & Number of rotor slots, $Z_{2}$ & 28 \\
3 & Length of the stator steel core, $L_{1}(\mathrm{~mm})$ & 100 \\
4 & Height of stator groove, $h_{01}(\mathrm{~mm})$ & 1 \\
5 & Height of rotor groove, $h_{02}(\mathrm{~mm})$ & 0.3 \\
6 & Selenium steel sheet number of stator, $n_{1}$ & 272 \\
7 & Selenium steel sheet number of rotor, $n_{2}$ & 277 \\
8 & Length of the rotor steel core, $L_{2}(\mathrm{~mm})$ & 102 \\
\hline
\end{tabular}

Table 2: The length of the rotating shaft discrete system.

\begin{tabular}{lccc}
\hline $\begin{array}{l}\text { Shaft } \\
\text { segment }\end{array}$ & Length $(\mathrm{mm})$ & $\begin{array}{c}\text { Outer diameter } \\
(\mathrm{mm})\end{array}$ & $\begin{array}{c}\text { Inner diameter } \\
(\mathrm{mm})\end{array}$ \\
\hline 1 & 20 & 45 & 32 \\
2 & 16 & 45 & 32 \\
3 & 10 & 45 & 33 \\
4 & 16 & 45 & 32 \\
5 & 5 & 53 & 32 \\
6 & 151 & 47 & 30 \\
7 & 15 & 40 & 30 \\
8 & 8 & 40 & 30 \\
9 & 15 & 40 & 30 \\
10 & 10 & 40 & 33 \\
\hline
\end{tabular}

arranged in an orderly manner and the magnetic force line closes quickly, and the eddy current effect of the stator and rotor is obvious. The magnetic density fluctuation frequency of the ceramic reverse magnetic material is less than that of the metal paramagnetic material. The magnetic density distribution of the ceramic spindle is less than that of the metal spindle.

Figure 7(a) shows the maximum magnetic density of the ceramic spindle stator pole gets to $7.5 \mu \mathrm{T}$ at $0.047 \mathrm{~s}$, which is less than the maximum magnetic density that the metal spindle gets to $(17.5 \mu \mathrm{T}$ at $0.033 \mathrm{~s})$. The maximum magnetic density of the ceramic spindle is lower than that of metal spindle. The variable frequency current of the spindle changes the flux direction of the rotor flux chain. Figure 7(b) shows the magnetic density distribution trend of spindle shell which the ceramic spindle shell can get to $0.011 \mu \mathrm{T}$ at $0.0232 \mathrm{~s}$ and the metal spindle gets to $(0.157 \mu \mathrm{T}$ at $0.0218 \mathrm{~s}$ ) are similar to the stator pole. The magnetic density of shell is much smaller than stator pole due to the increasing distance, which results in loss of magnetic lines.

According to Lenz's law, the molecular magnetic moment in the ceramic magnetic medium is stored in the opposite direction of the external magnetic field. The reverse induction electromotive force is generated and an additional antimagnetic field phenomenon occurs. The change speed magnetic density of the stator magnetic pole slows down. The magnetic density amplitude at the magnetic pole decreases; the metal spindle has the variable frequency current of the spindle that is consistent with the rotor flux chain. The magnetic field density of the magnetic pole increases under the paramagnetic action. 


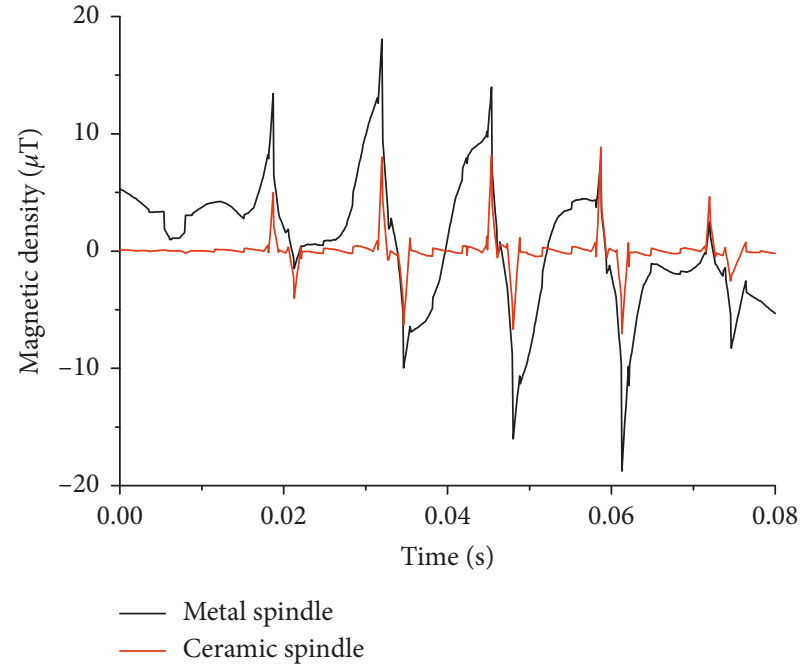

(a)

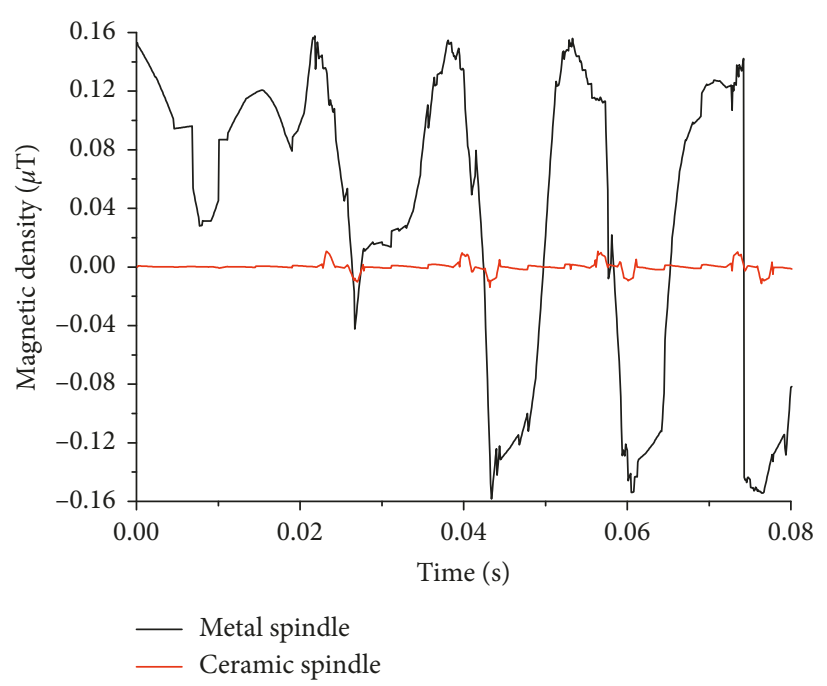

(b)

Figure 7: Magnetic density of the metal and ceramic spindles at $18000 \mathrm{rpm}$ : (a) the stator pole of spindle; (b) the shell of spindle.

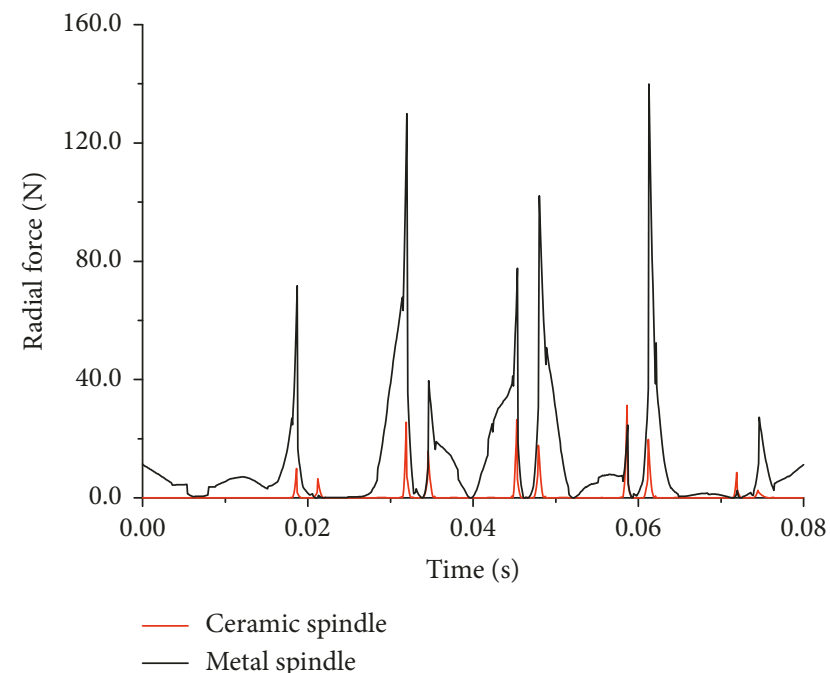

Figure 8: Radial force of the metal and ceramic spindles at $18000 \mathrm{rpm}$.

Based on the magnetic density values of the metal and ceramic spindles, the radial forces of the metal and ceramic spindles can be calculated by substituting it in formula (24). Figure 8 shows that the maximum unbalanced radial force of the metal spindle can reach $139.96 \mathrm{~N}$ at $0.048 \mathrm{~s}$. The maximum unbalanced radial force of the ceramic spindle at $0.025 \mathrm{~s}$ can reach $25.6 \mathrm{~N}$. The radial force distribution of the whole ceramic spindle is smaller than that of metal spindle. The radial force fluctuation of the ceramic spindle is less than that of metal spindle. The magnetization rate of the selenium metal shaft is different with the $40 \mathrm{Cr}$ metal rotor. The unevenness in magnetization is the main reason that the radial force of the metal spindle fluctuates so greatly.

The eccentric radial force of the ceramic spindle is shown in Figure 9. The radial force of the spindle points to the minimum air gap between the stator and rotor, and the air

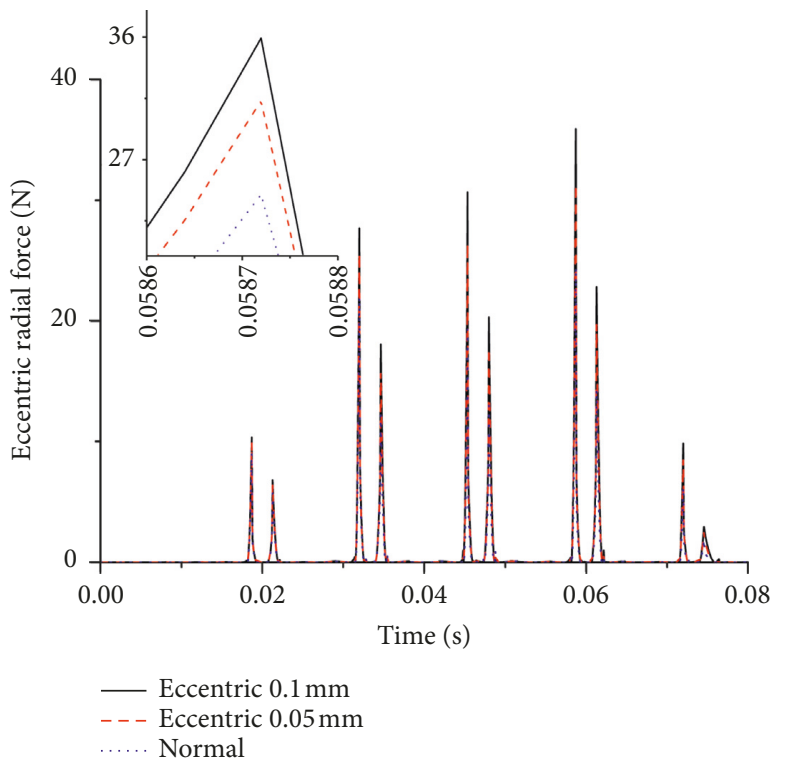

FIgURE 9: Eccentric radial force of the ceramic spindle at $18000 \mathrm{rpm}$.

gap directly affects the radial force of the spindle. The radial force of the air gap eccentricity and the magnetic field density of the stator and rotor increase with the increase in air gap eccentricity. The normal air gaps of a 170HT30 metal spindle and $170 \mathrm{SD} 30$ ceramic spindle are $0.3 \mathrm{~mm}$. The radial force of the uneccentric air gap of the ceramic spindle is $24.4256 \mathrm{~N}$, and the radial force of $0.05 \mathrm{~mm}$ is $31.3097 \mathrm{~N}$. The maximum eccentric air gap reaches $0.1 \mathrm{~mm}$. The minimum air gap between the stator and rotor is only $0.2 \mathrm{~mm}$, at which the maximum radial force at the minimum air gap is $35.9246 \mathrm{~N}$. The radial force of electromagnetic imbalance is $50 \%$ higher than that of a noneccentric electromagnetic force. Eccentricity causes uneven air gap distribution and leads to a rapid increase in magnetic field density at the 
magnetic poles. A small air gap leads to a rapid increase in bypass flux density, rapidly forming closed magnetic lines of force. Diffusion of the magnetic field density is inhibited, and the peak magnetic pole is rapidly increased. The radial force has a great impact on the vibration of spindle. The eddy current phenomenon inside the stator and rotor is accelerated, the air gap magnetic density is inhibited, and the skin effect gradient is increased. Compared with a normal air gap, the magnetic pole amplitude of an eccentric air gap decreases significantly to the surrounding area, which is the main factor why the fluctuation degree of eccentric radial force is greater than that of normal operation.

3.2. Spindle-Bearing Dynamic Stiffness Effect. According to formulas (8) and (9) for raceway control theory of a spindlebearing system, the bearing correction coefficient is adjusted for the characteristics of a ceramic $\mathrm{Si}_{3} \mathrm{~N}_{4}$ bearing to establish a ceramic bearing model and a metal bearing model, respectively. The conditions of simulation are radial dynamic stiffness, gyroscopic moment, and inner and outer ring contact angles of the bearing calculated under the working conditions of a preload force of $300-500 \mathrm{~N}$ and speed of 3000-30000 rpm.

Figure 10 shows the influence of preload change on the radial dynamic stiffness of metal and ceramic bearings. The radial stiffness of the rotating speed bearing is approximately decreasing with an increase in speed and increasing with an increase in pretension. At the same time, the preload force can hinder the change of radial stiffness with velocity. The radial stiffness of the ceramic bearing is greater than that of the metal bearing. Figure 10(b) shows that the radial dynamic stiffness of the ceramic bearing reaches a maximum value of $2.252 \times 10^{8}$ under the working condition of a speed of $3000 \mathrm{rpm}$ and preload of $500 \mathrm{~N}$. Since the speed increase is affected by the centrifugal force of the rolling body, there is a nonmonotone decreasing law and the bearing stiffness has a "softening effect." The trend of metal bearings is the same as that of ceramic bearings at low speed and medium speed.

Under the condition of low speed and a large preload, Figure 10 (a) shows that the maximum value is $1.676 \times 10^{8}$. The turning point occurs when the speed exceeds $18000 \mathrm{rpm}$ under the action of the centrifugal force of the bearing. When the metal bearing speed is above $18000 \mathrm{rpm}$, the centrifugal force of the rolling body increases. The radial dynamic stiffness of metal bearings is greatly affected by the contact expansion deformation between the outer ring raceway and ball, and the contact angle with the external load decreases to lead to the bearing operation mechanism changing. The decrease in inner ring stiffness is less than the increase in outer ring stiffness. For the ceramic bearing, the characteristics of large material stiffness lead to the deformation of outer ring channel being small. The change degree of the decrease in the stiffness of the inner ring is greater than that of the increase in the stiffness of the outer ring. The radial stiffness of the ceramic bearing tends to decrease, and the bearing stiffness softening effect is obvious.

Figure 11 shows the comparison of different parameters of metal and ceramic bearings. The bearing operating speed range is $3000-30000 \mathrm{rpm}$, and the preload force is $400 \mathrm{~N}$. Figure 11(a) shows that as the friction torque and centrifugal force between the ball and the bearing raceway with the ceramic bearing and metal bearing speed increase, the gyroscopic moment increases significantly and shows a rapid upward trend. The high stiffness and low density of the ceramic bearing are the main reasons that the gyroscopic torque of the ceramic bearing is less than that of metal bearing. Figure 11(b) shows that as the outer ring contact angle of the metal and ceramic bearings increases with the speed, the inner ring contact angle decreases. The inner and outer rings of the high-speed rotating bearing are affected by the centrifugal force of the ball and the gyroscopic moment, which is changing the contact mechanism. The contact stress and amount of deformation between the bearing ball and the raceway of inner and outer ring are changed.

The metal bearing outer ring contact angle is increased from $15.44^{\circ}$ to $22.82^{\circ}$. The ceramic bearing outer ring contact angle is increased from $15.04^{\circ}$ to $19.77^{\circ}$. The contact angle of the metal bearing changes more with the increase of speed than that of the ceramic bearing. The ceramic bearing inner ring contact angle reduces to $4.63^{\circ}$ and is $52 \%$ of the reduction in the contact angle of the inner ring of the metal bearing. There is an approximate linear relationship between the centrifugal force of the bearing ball, gyroscopic moment, and the contact angle of the bearing outer ring raceway. The ball mass of the ceramic bearing being smaller than that of metal bearing is the main factor that means the centrifugal force and gyroscopic moment of the ceramic bearing are less than those of metal bearing. At the same time, the high stiffness of the ceramic bearing reduces the inner and outer ring raceway deformation and improves the dynamic performance of the bearing.

\section{Experimental Verification and Analysis}

4.1. Test Magnetic Field and Vibration of Spindle. Oil and gas lubrication is adopted for spindle-bearing lubrication under the test conditions. The experimental conditions are that the input oil is $5 \mathrm{~mL} / \mathrm{h}$, air pressure is $0.25 \mathrm{MPa}$, cooling water temperature is $18^{\circ}$, the cooling water flow rate is $5 \mathrm{~L} / \mathrm{min}$, and bearing preload force is $400 \mathrm{~N}$. The speed range, for which each increment is $1000 \mathrm{rpm}$, is from $9000 \mathrm{rpm}$ to $18000 \mathrm{rpm}$. A Japan HIOKI FT3470-52 magnetic field tester was used to conduct a magnetic density test of the spindle magnetic field for a 170SD30 ceramic spindle and 170HT30 metal spindle. Figure 12(a) shows the magnetic field density test of a ceramic spindle shell measured on two positions by an electromagnetic field tester. A and B points are, respectively, stator position and bearing position on the spindle shell surface which are shown in Figure 12(a). A German Polytec OFV-505 contactless laser vibration measurement system was used to measure the spindle end vibration based on the laser Doppler effect. Figure 12(b) shows the spindle end vibration velocity test of the ceramic spindle. The basic parameters of the 170SD30 ceramic spindle and 170HT30 metal spindle are shown in Table 3. 


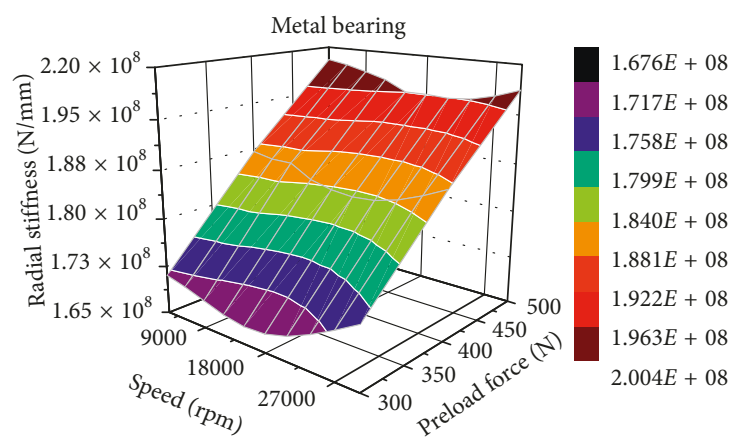

(a)

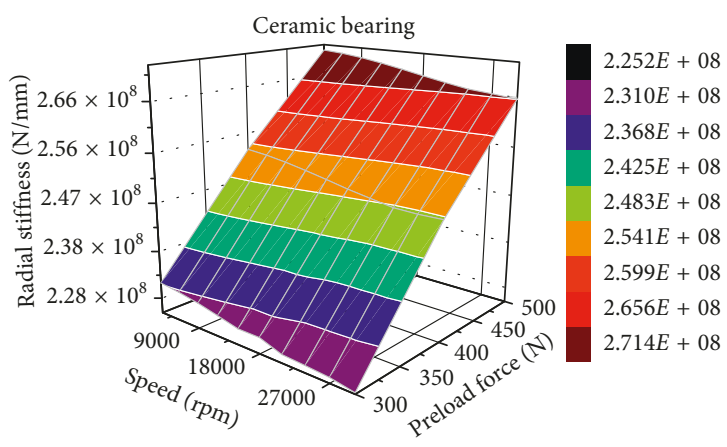

(b)

Figure 10: Comparison of preload force of radial dynamic stiffness of (a) metal bearing and (b) ceramic bearing.

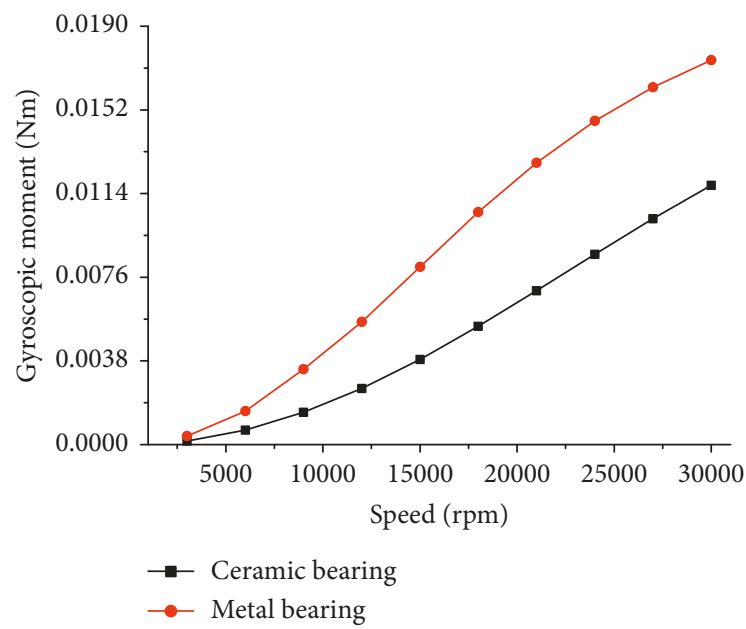

(a)

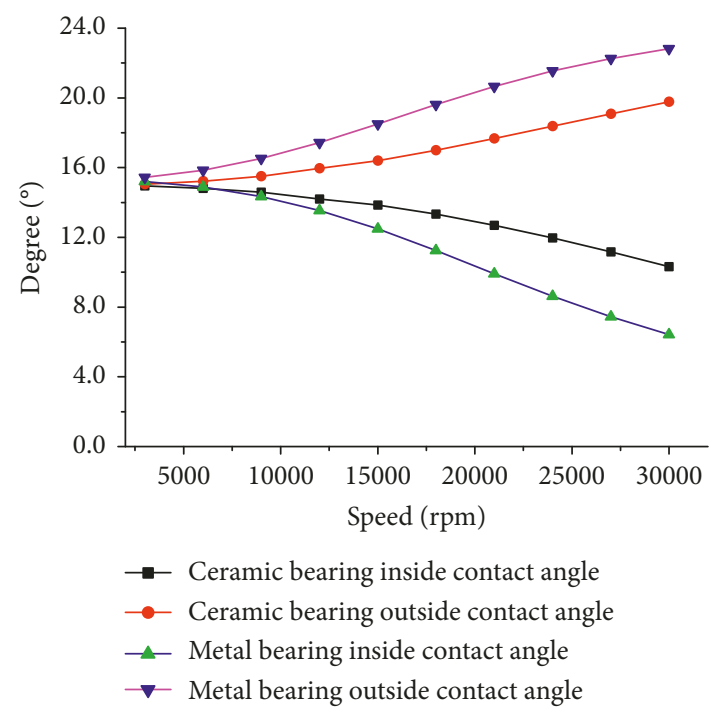

(b)

FIGURE 11: The comparison of different parameters of metal and ceramic bearings: (a) gyroscopic moment; (b) inner and outer ring contact angle.

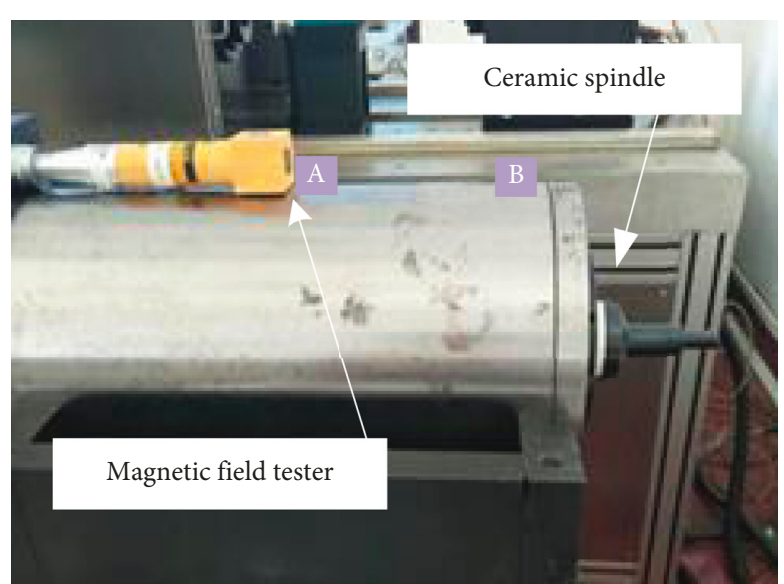

(a)

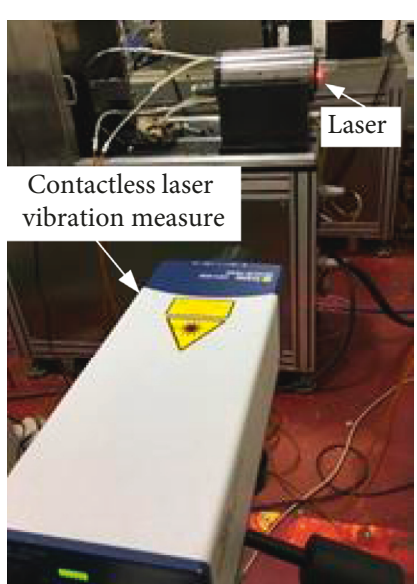

(b)

FIGURE 12: Ceramic spindle: (a) magnetic field and (b) vibration test. 
TABLE 3: Basic parameters of spindle.

\begin{tabular}{lcc}
\hline Basic parameters of spindle & $\begin{array}{c}170 \mathrm{SD} 30 \text { ceramic } \\
\text { spindle }\end{array}$ & $\begin{array}{c}170 \mathrm{HT} 30 \text { metal } \\
\text { spindle }\end{array}$ \\
\hline Rated speed (rpm) & 30000 & 30000 \\
Rated voltage (V) & 350 & 350 \\
Rated frequency (Hz) & 1000 & 1000 \\
Pole logarithm & 4 & 4 \\
Shaft material & $\mathrm{ZrO}_{2}$ ceramic & $40 \mathrm{Cr}$ steel \\
Bearing inner and & $\mathrm{ZrO}_{2}$ ceramic & $\mathrm{GCr} 15$ steel \\
outer ring material & $\mathrm{Si}_{3} \mathrm{~N}_{4}$ ceramic & GCr15 steel \\
Bearing ball material & & \\
\hline
\end{tabular}

4.2. Test Results and Analysis. The magnetic field density of the electric spindle is mainly concentrated in the magnetic pole of the stator and the radial shell region. The distance between the magnetic field density and magnetic poles is reversely proportional. The magnetic field density of the outer stator shell is less than that of the magnetic pole. The spindle shell is tested by an electromagnetic field tester that can reflect the variation law of the electromagnetic field density between fixed rotor magnetic poles.

Magnetic density comparisons of the ceramic and metal spindles in A and B positions are, respectively, shown in Figures 13(a) and 13(b). Under test conditions at $18000 \mathrm{rpm}$ to measure the magnetic density, A point of the metal and ceramic spindles, respectively, are $0.09475 \mu \mathrm{T}$ and $0.0089 \mu \mathrm{T}$; meanwhile, the B point are, respectively, $0.08231 \mu \mathrm{T}$ and $0.00823 \mu \mathrm{T}$. It can be clearly seen that the magnetic field density of the metal spindle shell is higher than that of ceramic spindle shell. The magnetic density of the B point is a little smaller than A point that is attributed to the loss of the magnetic induction line. The magnetic density error between the simulation and experiment of the ceramic and metal spindles in A point, respectively, is $0.0018 \mu \mathrm{T}$ and $0.01095 \mu \mathrm{T}$ and B point, respectively, is $0.00101 \mu \mathrm{T}$ and $0.00799 \mu \mathrm{T}$. The prediction of the magnetization model can be high, precisely verified through the comparisons with different positions.

The magnetic density of the spindle fluctuates under the action of an eddy current and reverse magnetism is shown in Figure 14. It shows the magnetic density time domain diagram of the ceramic spindle at $18000 \mathrm{rpm}$. It is consistent with the trend of magnetic density simulation diagram in Figure 7 , which proves the accuracy of the electromagnetic model.

Spindle vibration is mainly composed of mechanical vibration and electromagnetic vibration. The vibration velocity frequency domain diagram for the shaft end of ceramic and metal spindles at $18000 \mathrm{rpm}$ is shown in Figure 15. The amplitude of the metal spindle in the low-frequency region and high-frequency region is larger than that of the ceramic spindle in the frequency domain diagram. This shows that the radial force of electromagnetic imbalance, friction force between the bearing and rotating shaft, centrifugal force, and other factors in the spindle system are the components that influence the frequency distribution. One time the frequency of rotation mainly includes electromagnetic magnetic tension. Two times the frequency of rotation mainly includes the bearing centrifugal force and

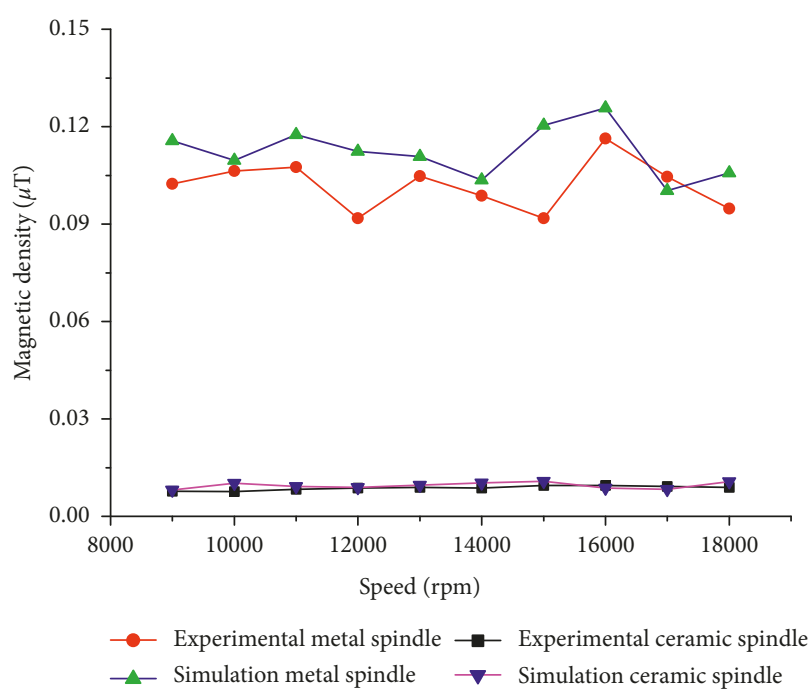

(a)

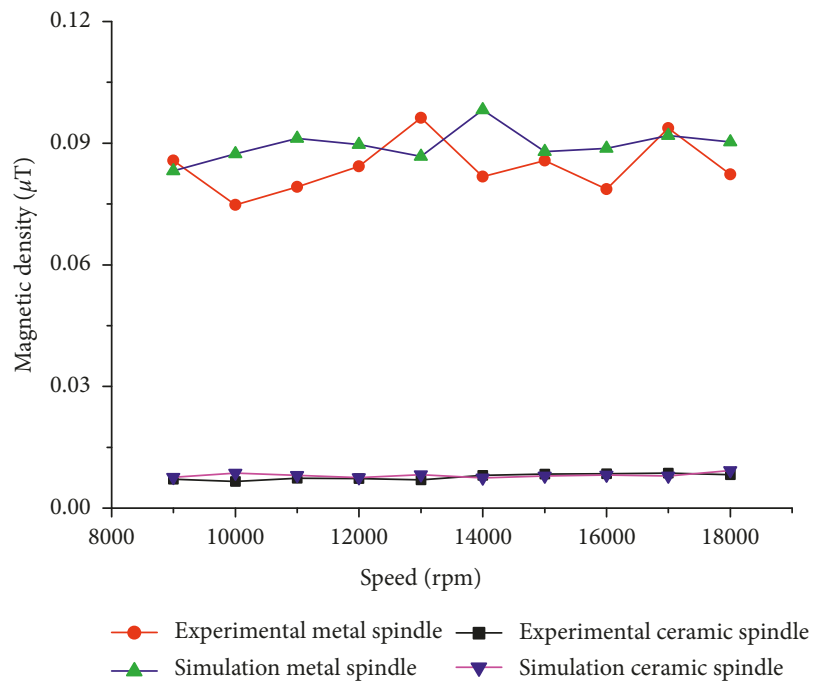

(b)

FIgURE 13: Magnetic density comparison of the ceramic and metal spindles. (a) A point: stator position on the spindle shell surface. (b) B point: bearing position on the spindle shell surface.

friction force excitation frequency. The energy of 1 time the frequency in the low-frequency region is greater than that in the middle- and high-frequency regions.

In Figure 15(a), the peak value of 1 time the ceramic spindle frequency is lower than that of 2 times the frequency. This shows that the electromagnetic radial force of the ceramic spindle has less influence on the vibration of the spindle than the Hertz force of the bearing. In Figure 15(b), the peak value of 1 time the frequency of the metal spindle is greater than that of 2 times the frequency of the metal spindle. It can be seen that the electromagnetic radial force of the metal spindle has a great impact on the vibration of the spindle. In Figures 15(a) and 15(b), the amplitude of 1 and 2 times the frequency of the metal spindle frequency domain with vibration shock energy is larger than that of the ceramic spindle frequency. The influence of vibration velocity on 


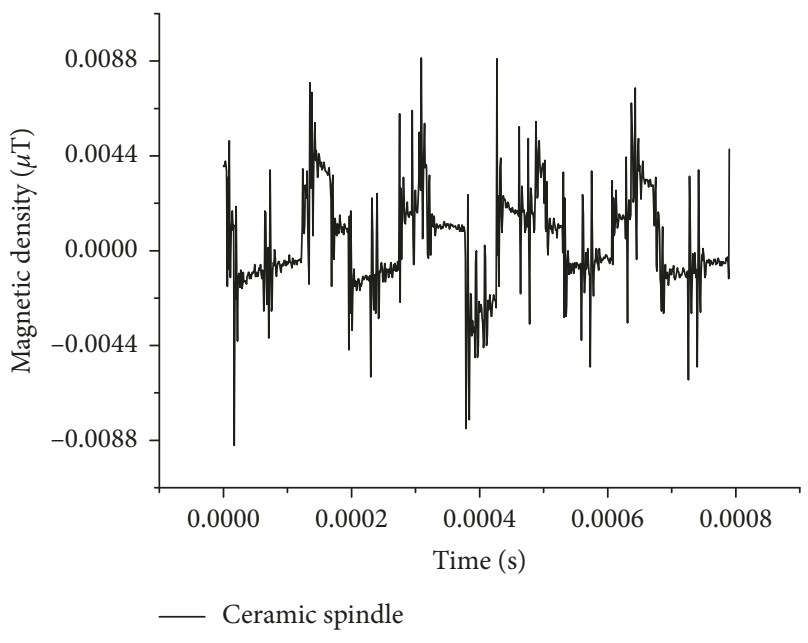

FIGURE 14: Magnetic density time domain diagram of the ceramic spindle at $18000 \mathrm{rpm}$.

metal spindle is more obvious. The influence of electromagnetic force and bearing force on the spindle is analyzed in the frequency domain. Under the working condition of $18000 \mathrm{rpm}$, the frequency domain is approximately $600 \mathrm{~Hz}$, so a frequency range within $1000 \mathrm{~Hz}$ is selected.

Figure 16 shows the comparison with the diagram of spindle end vibration velocity test. It can be seen that the vibration speed of the shaft end presents an increasing trend with the increase in rotating speed. The vibration speed of the shaft end of the metal spindle is higher than that of the ceramic spindle. This indicates that the Hertz contact force and centrifugal force caused by the change of rotation speed parameters are the main factor affecting the vibration.

As seen in Figure 16(a), without considering the dynamic characteristics of electromagnetic radial force, the simulation vibration velocity is roughly the same as the variation trend of spindle vibration. The vibration velocity of the shaft end calculated by the simulation is less than that of the test result, and there is a large error compared with the real value. The amplitude difference of vibration velocity between the metal spindle simulation and experiment is higher than that of the ceramic spindle.

From Figure 16(b), considering the dynamic characteristics of the traditional electromagnetic radial force, the simulation vibration speed of the shaft end of the metal spindle is slightly higher than the test result. However, the traditional model does not take into account the reverse magnetic effect of ceramics. The spindle end vibration amplitude calculated by theory is much larger than the test results, and the error even reaches $20 \%$ under the working condition of $14000 \mathrm{rpm}$, which cannot effectively reflect the vibration characteristics of the ceramic spindle.

From Figure 16(c), considering the magnetization effect, the simulation speed of electromagnetic radial force dynamics is in good agreement with the test results. The electromagnetic radial force calculated after the magnetization of the ceramic reverse magnetic effect and metal paramagnetic effect has a high accuracy in obtaining the vibration simulation speed. In particular, the calculation

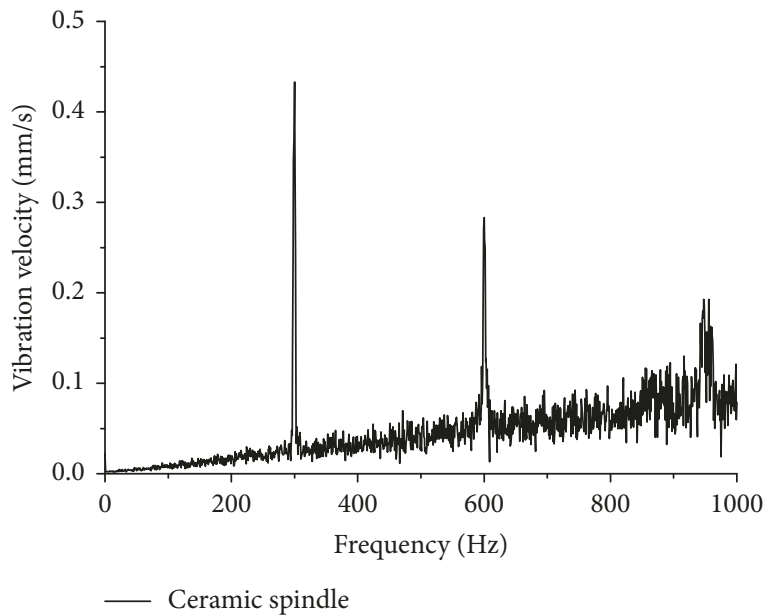

(a)

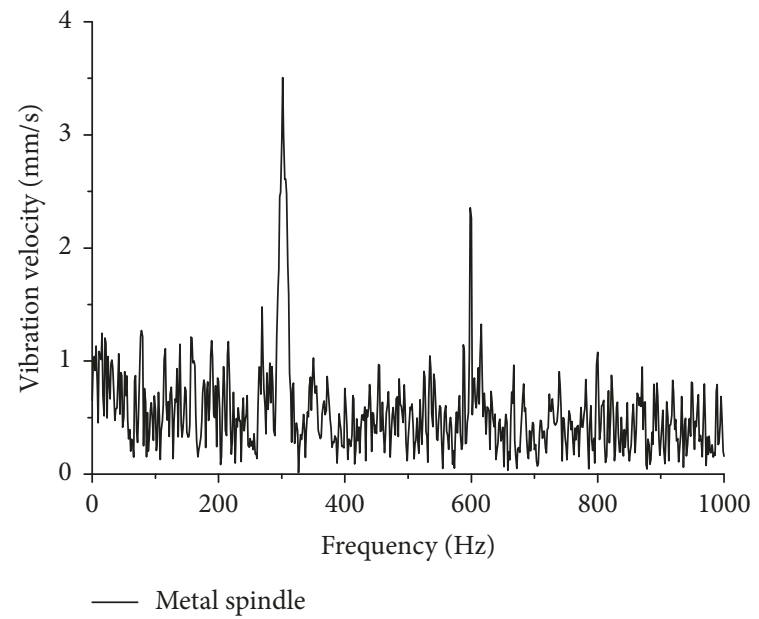

(b)

FIgURE 15: Vibration velocity frequency domain diagram at the spindle shaft end at $18000 \mathrm{rpm}$ : (a) ceramic spindle; (b) metal spindle.

accuracy of the ceramic spindle is greatly improved. Under the test condition of $18000 \mathrm{rpm}$, the vibration speed at the shaft end of the metal spindle was measured as $23.4 \mathrm{~mm} / \mathrm{s}$, and that at the shaft end of the ceramic spindle was measured as $18.65 \mathrm{~mm} / \mathrm{s}$. The simulation accuracy of the vibration velocity of the metal and ceramic spindle ends is reduced from the traditional dynamic magnetic model error values of $2.4 \%$ and $16.1 \%$ to $1.4 \%$ and $1.17 \%$, respectively. It shows that the experimental results are consistent with the theoretical results, and the magnetization theoretical model can effectively simulate the vibration speed under different working conditions.

\section{Conclusion}

The electromagnetic coupling dynamics model considered reverse magnetic effect which can be used to predict magnetic field and vibration on the ceramic spindle is presented. The magnetic density of the ceramic spindle affected by reverse magnetic effect, Hertz contact force of the bearing, 


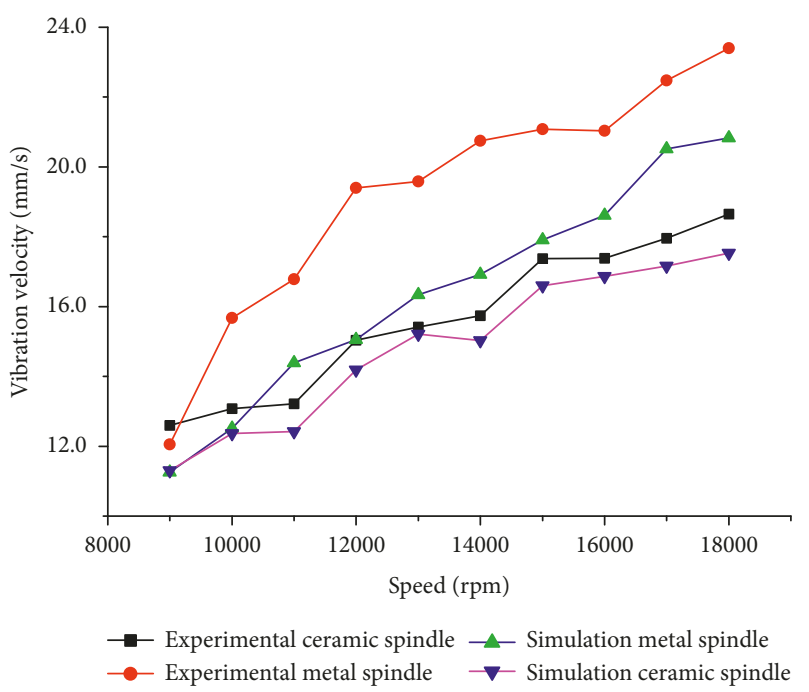

(a)

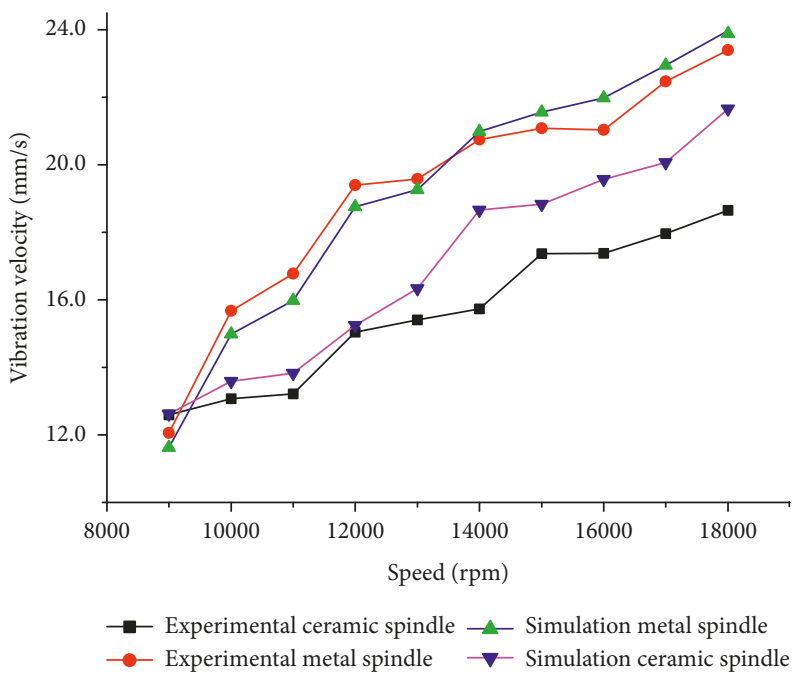

(b)

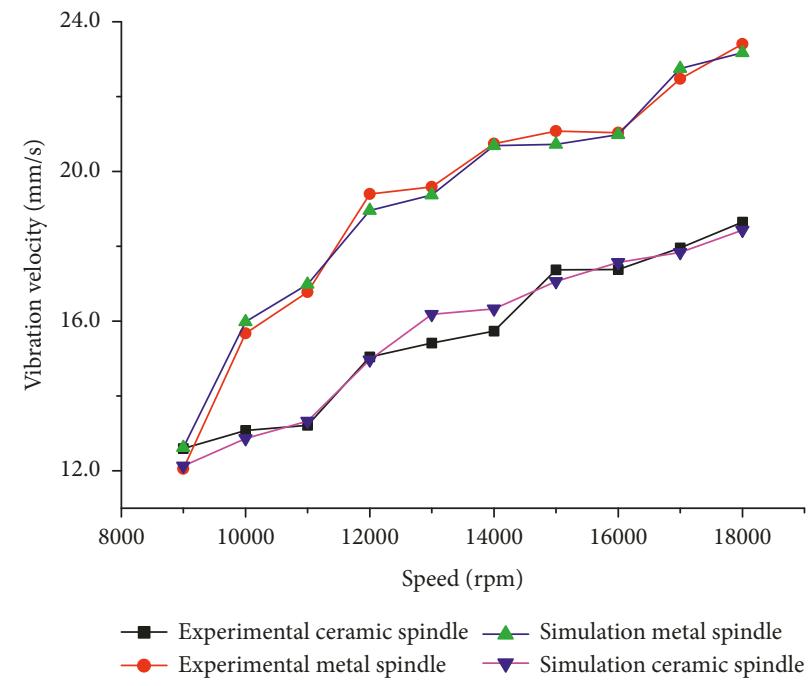

(c)

FIGURE 16: Vibration velocity in the time domain between ceramic and metal spindles under $400 \mathrm{~N}$ bearing preload. (a) Without considering the dynamic characteristics of electromagnetic radial force comparison. (b) Considering the dynamic characteristics of traditional electromagnetic radial force comparison. (c) Considering the dynamic characteristics of the magnetization effect.

and Maxwell stress of the rotor is analyzed. Furthermore, the effects of the rotational speed, bearing stiffness, and the magnetic radial force on the dynamic characteristics of highspeed spindle are studied in detail. The following conclusions are drawn:

(1) The actual working conditions of a high-speed rotating eccentric electromagnetic unbalanced radial force and bearing contact force excitation of a spindle system are studied. The coupling dynamics model considered reverse magnetic effect of a ceramic spindle was established. The simulation results are basically consistent with the experimental results. A more realistic simulation of speed and bearing contact force on spindle vibration has verified that the prediction of magnetization dynamic magnetic coupling model has high accuracy.
(2) Based on the Maxwell principle, the electromagnetic model of a ceramic spindle is established for the reverse magnetic characteristics of ceramic materials. The characteristics of ceramic reverse magnetic materials, electromagnetic magnetic density, and radial force generated by various working conditions are studied. The electromagnetic radial force of the ceramic spindle is smaller than that of the metal spindle. Compared with the experimental results, the simulation results of magnetization dynamic magnetic coupling model have smaller errors.

(3) The basic characteristics of a bearing, such as centrifugal force, gyroscopic moment, and contact angle of the inner and outer ring, are coupled together to establish a ceramic high-speed angular contact ball bearing model. It is calculated that the dynamic 
stiffness of a metal bearing is less than that of a ceramic bearing. With the increase in speed, the stiffness of the ceramic bearing appears to "soften." When the rotating speed reaches more than $18000 \mathrm{rpm}$, the centrifugal force of the metal bearing, gyroscopic moment, and ball contact angle of the inner ring increase significantly. The coupling effect changes the bearing operating mechanism to lead the stiffness to rise.

\section{Nomenclature}

$T: \quad$ Kinetic energy of spindle segment

$W$ : Damping work of spindle segment

$V: \quad$ Deformation energy of spindle segment

$S: \quad$ Cross-sectional area

$\theta_{z}: \quad$ Rotation angle of the $x-y$ section

$\theta_{y}$ : $\quad$ Rotation angle of the $x-z$ section

$a$ : Inner diameter of rotating shaft cross section

$b$ : $\quad$ Outer diameter of rotating shaft cross section

A: Magnetic potential

E: $\quad$ Elasticity modulus

$v$ : Poisson ratio

$\Omega: \quad$ Spindle speed

$G$ : $\quad$ Shear modulus

$\rho: \quad$ Material density

$W_{\mathrm{s}}: \quad$ Stator winding circles

$K_{\mathrm{s}}: \quad$ Shear coefficient

$L: \quad$ Length of spindle beam element

$\delta$ : $\quad$ Total deformation of bearing ball with inner and outer ring raceway

$\lambda_{\mathrm{o} j}: \quad$ Friction coefficient of outer ring

$\lambda_{\mathrm{i} j}$ : $\quad$ Friction coefficient of inner ring

$r: \quad$ Radius

$\tan \beta$ : Control correction factor of bearing

$Q$ : $\quad$ Contact stress between bearing ring and ball

$K$ : $\quad$ Contact stiffness of bearing ball with ring raceway

$M$ : $\quad$ Moment of inner ring

$\mu_{0}: \quad$ Air permeability

$B$ : $\quad$ Magnetic density

$\Delta l: \quad$ Stator and rotor contact length

$\xi$ : $\quad$ Electrical angle

$\gamma$ : Offset angle of rotor

$H$ : Magnetic intensity vector of the spindle

$\omega$ : Fundamental wave rotates angular velocity

$d: \quad$ The distance

$D_{2 \mathrm{v}}$ : Damping coefficient

$\theta$ : $\quad$ Rotation angle of rotor

$\varphi_{0}$ : $\quad$ Power factor angle

$K_{\mathrm{dp} 0}$ : Winding coefficient

$j$ : $\quad$ Current density

\footnotetext{
Subscript

i: Inner ring

o: Outer ring

bc: Ball center

p: Pitch

jk: Gyroscopic
}

$u$ : Rotor harmonic frequency

$v$ : Stator harmonic frequency

b: Bearing ball

0: Fundamental wave

ok: Between bearing outer ring and ball

ik: Between bearing inner ring and ball

$z$ r: Radial direction of bearing

$z$ : Bearing

ap: Preload of bearing

a: Axial direction

s: Stator

r: Rotor

$q$ : The eccentricity of air gap

c: $z$ point to the center of the rotor

$\mathrm{m}$ : magnetization.

\section{Data Availability}

The data used to support the findings of this study are available from the corresponding author upon request.

\section{Conflicts of Interest}

The authors declare that they have no conflicts of interest.

\section{Acknowledgments}

This work was supported by the National Natural Science Foundation of China (Grant numbers 51675353, 51705341, and 51805337), the innovation team project about the Ministry of Education of China (Grant number IRT-15R45), and the Programme of Introducing Talents of Discipline to Universities (Grant number D18017).

\section{References}

[1] F. Forestier, V. Gagnol, P. Ray, and H. Paris, "Model-based cutting prediction for a self-vibratory drilling head-spindle system," International Journal of Machine Tools \& Manufacture, vol. 52, pp. 59-68, 2012.

[2] E. Abele, Y. Altintas, and C. Brecher, "Machine tool spindle units," CIRP Annals, vol. 59, no. 2, pp. 781-802, 2010.

[3] B. Wang, W. Sun, K. Xu, J. Zhang, and B. Wen, "The nonlinear stability prediction and FEM modeling of high-speed spindle system with joints dynamic characteristics," Shock and Vibration, vol. 2014, Article ID 153504, 12 pages, 2014.

[4] A.-C. Lee and T.-D. Hoang, "Coupled lateral and torsional vibrations of the micro-drilling spindle systems," The International Journal of Advanced Manufacturing Technology, vol. 87, no. 5-8, pp. 2063-2079, 2016.

[5] A. Zivkovic, M. Zeljkovic, S. Tabakovic, and Z. Milojevic, "Mathematical modeling and experimental testing of highspeed spindle behavior," International Journal of Advanced Manufacturing, vol. 77, no. 5-8, pp. 1071-1086, 2015.

[6] G. Hu, W. Gao, Y. Chen et al., "An experimental study on the rotational accuracy of variable preload spindle-bearing system," Advances in Mechanical Engineering, vol. 10, no. 5, 2018.

[7] C. Hong, S. Ibaraki, and A. Matsubara, "Influence of positiondependent geometric errors of rotary axes on a machining test of cone frustum by five-axis machine tools," Precision Engineering, vol. 35, no. 1, pp. 1-11, 2011. 
[8] M. Postel, O. Özsahin, and Y. Altintas, "High speed tooltip FRF predictions of arbitrary tool-holder combinations based on operational spindle identification," International Journal of Machine Tools and Manufacture, vol. 129, pp. 48-60, 2018.

[9] W. Dou, X. He, and B. Tang, "Stability analysis of machine tool spindle under uncertainty," Advances in Mechanical Engineering, vol. 8, no. 5, 2016.

[10] X. Wang, Y. Guo, and T. Chen, "Measurement research of motorized spindle dynami stiffness under high speed rotating," Shock and Vibration, vol. 2015, Article ID 284126, 11 pages, 2015.

[11] J. Vyroubal, "Compensation of machine tool thermal deformation in spindle axis direction based on decomposition method," Precision Engineering, vol. 36, no. 1, pp. 121-127, 2012.

[12] J. Yang, H. Shi, B. Feng, L. Zhao, C. Ma, and X. Mei, “Thermal error modeling and compensation for a high-speed motorized spindle," The International Journal of Advanced Manufacturing Technology, vol. 77, no. 5-8, pp. 1005-1017, 2015.

[13] N. Grossi, "Accurate and fast measurement of specific cutting force coefficients changing with spindle speed," International Journal of Precision Engineering and Manufacturing, vol. 18, no. 8, pp. 1173-1180, 2017.

[14] U. Werner, "Rotordynamic model for electromagnetic excitation caused by an eccentric and angular rotor core in an induction motor," Archive of Applied Mechanics, vol. 83, no. 8, pp. 1215-1238, 2013.

[15] J. Liu, T. Lai, and X. Chen, "Dynamics analysis of unbalanced motorized spindles supported on ball bearings," Shock and Vibration, vol. 2016, Article ID 2787524, 10 pages, 2016.

[16] M. Donát, "Computational modelling of the unbalanced magnetic pull by finite element method," Procedia Engineering, vol. 48, pp. 83-89, 2012.

[17] J. Xul, X. Zheng, J. Zhang, and X. Liu, "Vibration characteristics of unbalance response for spindle system," Procedia Engineering, vol. 174, pp. 331-340, 2017.

[18] A. K. Verma, S. Sarangi, and M. H. Kolekar, "Experimental investigation of misalignment effects on rotor shaft vibration and on stator current signature," Journal of Failure Analysis and Prevention, vol. 14, no. 2, pp. 125-138, 2014.

[19] J. A. Oliver, G. Guerrero, and J. Goldman, "Ceramic bearings for electric motors: eliminating damage with new materials," IEEE Industry Applications Magazine, vol. 23, no. 6, pp. 14-20, 2017.

[20] V. Mironov, E. Indriksone, I. Beljaeva, V. Glushchenkov, and A. Korjakins, "Combined magnetic pulsed compaction of oxide ceramic powder materials," Key Engineering Materials, vol. 674 , pp. 88-93, 2016.

[21] H. A. Khazdozian, R. L. Hadimani, and D. C. Jiles, "Development of rare earth free permanent magnet generator using Halbach cylinder rotor design," Renewable Energy, vol. 112, pp. 84-92, 2017.

[22] D.-H. Kim and C.-M. Lee, "Development of an automatic variable preload device using uniformly distributed eccentric mass for a high-speed spindle," International Journal of Precision Engineering and Manufacturing, vol. 18, no. 10, pp. 1419-1423, 2017.

[23] Y. Cao and Y. Altintas, "A general method for the modeling of spindle-bearing systems," Journal of Mechanical Design, vol. 126, no. 6, pp. 1089-1104, 2004.

[24] V.-T. Than and J. H. Huang, "Nonlinear thermal effects on high-speed spindle bearings subjected to preload," Tribology International, vol. 96, pp. 361-372, 2016.
[25] C.-B. Gan, Y.-H. Wang, S.-X. Yang, and Y.-L. Cao, "Nonparametric modeling and vibration analysis of uncertain Jeffcott rotor with disc offset," International Journal of Mechanical Sciences, vol. 78, pp. 126-134, 2014.

[26] S. Sung, G. Jang, and K. Kang, "Noise and vibration due to rotor eccentricity in a HDD spindle system," Microsystem Technologies, vol. 20, no. 8-9, pp. 1461-1469, 2014.

[27] H. Im, H. H. Yoo, and J. Chung, "Dynamic analysis of a BLDC motor with mechanical and electromagnetic interaction due to air gap variation," Journal of Sound and Vibration, vol. 330, no. 8, pp. 1680-1691, 2011.

[28] V. P. Legaev and L. K. Generalov, "Control system for a machine-tool drive with compensation of spindle eccentricity," Russian Engineering Research, vol. 32, no. 5-6, pp. 430432, 2012.

[29] C. Ma, S. Zuo, R. He, and L. He, "Radial electromagnetic force wave analysis of permanent magnet synchronous motor for electric vehicle," Advanced Materials Research, vol. 211-212, pp. 948-952, 2011. 


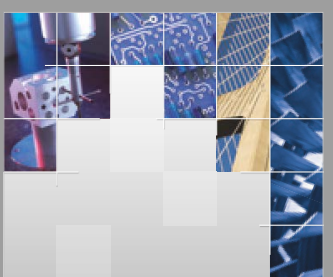

\section{Enfincering}
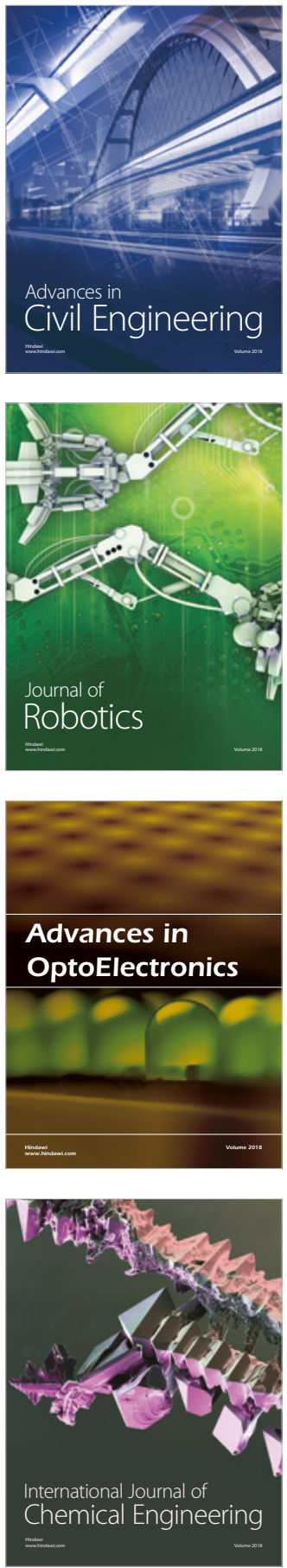

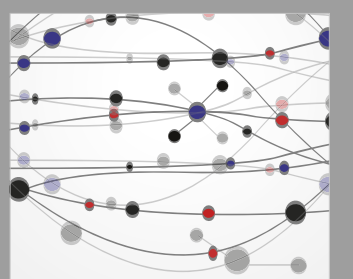

\section{Rotating \\ Machinery}

The Scientific World Journal

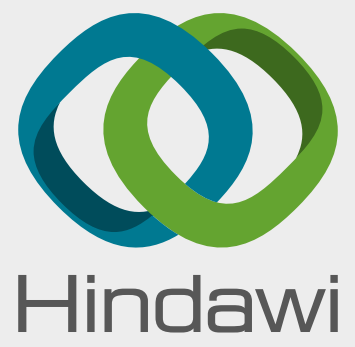

Submit your manuscripts at

www.hindawi.com
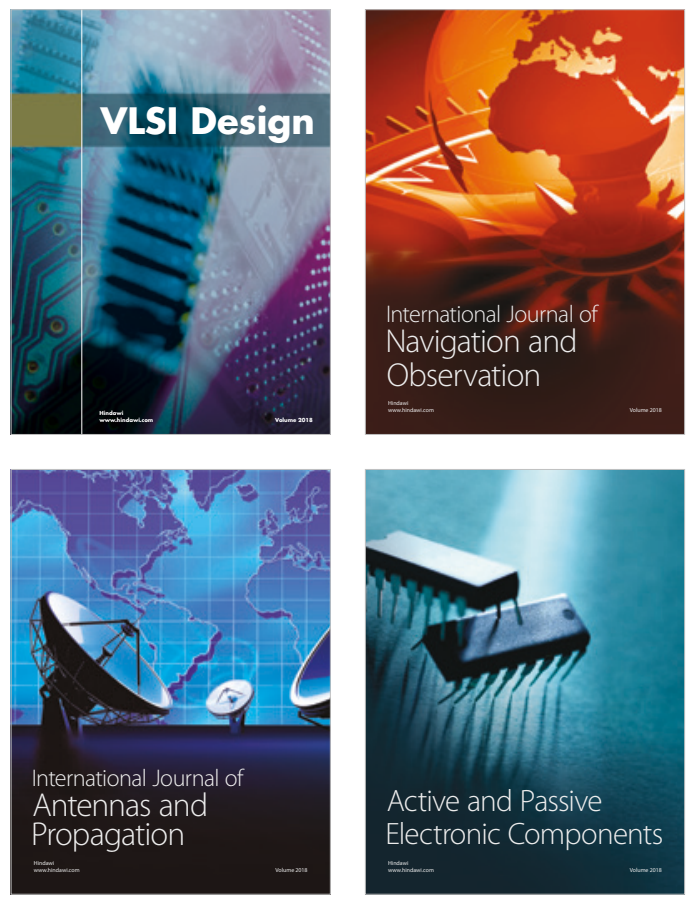
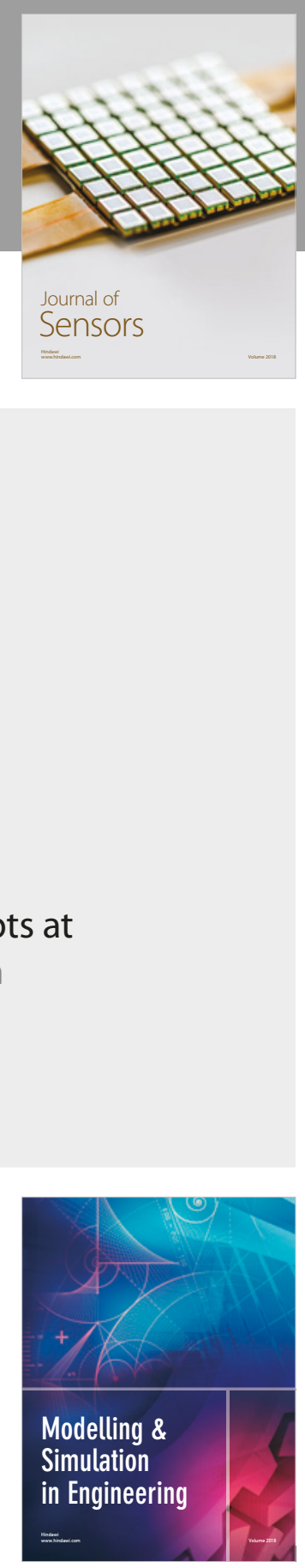

\section{Advances \\ Multimedia}
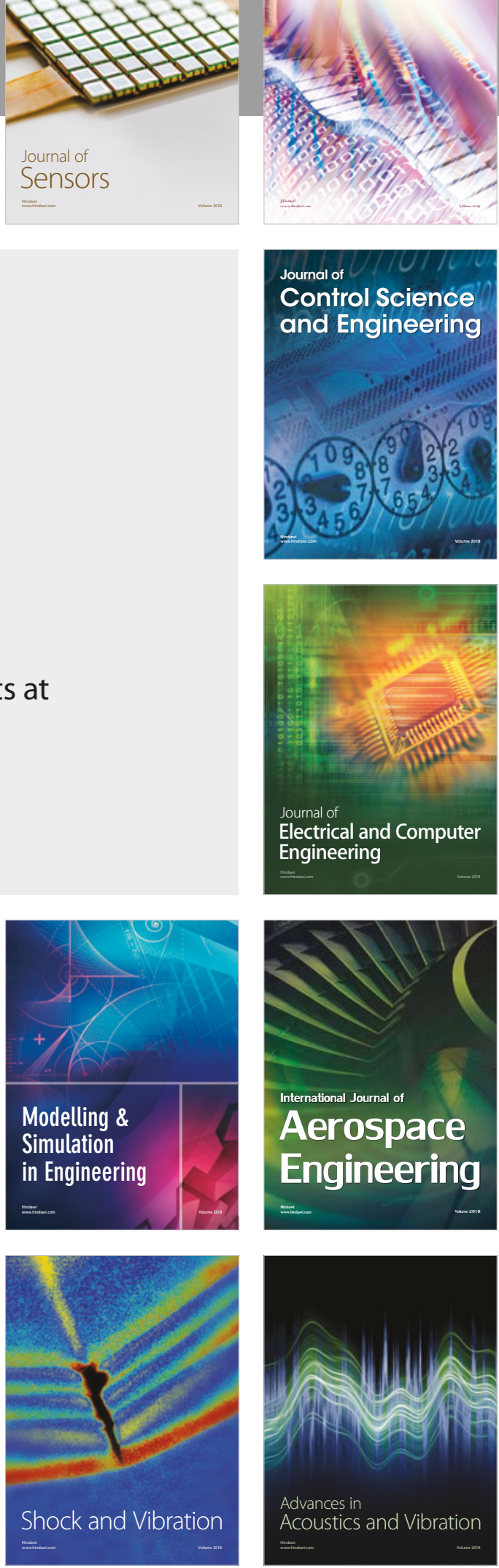